Meinert, Franz/Geerds, Friedrich (1976): Vernehmungstechnik, Lübeck: Verlag für polizeiliches Fachschrifttum

Ohe, Werner von der (1987): Interethnische Beziehungen als Dolmetscherarbeit, in: ders. (Hg.), Kulturanthropologie, Berlin: Duncker \& Humblot, S. 401-420

Plessner, Helmuth (1979): Mit anderen Augen, in: ders., Zwischen Philosophie und Gesellschaft, Frankfurt/M.: Suhrkamp, S. 233-248

Renn, Joachim (2006). Übersetzungsverhältnisse. Perspektiven einer pragmatischen Gesellschaftstheorie. Weilerswist: Velbrück

Renn, Joachim (1999): Der Tod des Kapitän Cook. Zur Pragmatik sozialer Integration am Beispiel einer interkulturellen Begegnung, in: Handlung, Kultur, Interpretation 8, S. 5-26

Sanjek, Roger (Hg.) (1991): Fieldnotes: The Making of Anthropology, Ithaca, London: Cornell University Press

Schröer, Norbert (1997): Wissenssoziologische Hermeneutik, in: Ronald Hitzler/Honer, Anne (Hg.), Sozialwissenschaftliche Hermeneutik, Opladen: Leske+Budrich, S. 109-129

Schröer, Norbert (2002): Verfehlte Verständigung? Kommunikationssoziologische Fallstudie zur interkulturellen Kommunikation. Konstanz: UVK

Schütz, Alfred (1971): Symbol, Wirklichkeit und Gesellschaft, in: ders., Gesammelte Aufsätze 1, Den Haag: Martinus Nijhoff, S. 331-411

Soeffner, Hans-Georg (1989): Auslegung des Alltags - Der Alltag der Auslegung. Frankfurt/M.: Suhrkamp.

Stagl, Justin (1980): Szientistische, hermeneutische und phänomenologische Grundlagen der Ethnologie, in: Wolfdietrich SchmiedKowarzik/Stagl, Justin (Hg.), Grundfragen der Ethnologie, Berlin: Dietrich Reimer, S. 1-38

Tedlock, Dennis (1985): Die analogische Tradition und die Anfänge einer dialogischen Anthropologie, in: Trickster 12/13, S. 62-74

Turner, Victor (1995): Vom Ritual zum Theater. Der Ernst des menschlichen Spiels. Frankfurt/M.: Suhrkamp

Tyler, Steven (1993): Zum »Be-/Abschreiben« als »Sprechen für«. Ein Kommentar, in: Eberhard Berg/Fuchs, Martin (Hg.), Kultur, soziale Praxis, Text. Die Krise der ethnographischen Repräsentation, Frankfurt/M.: Suhrkamp, S. 288-296

Tyler, Steven (1991): Das Unaussprechliche. Ethnographie, Diskurs und Rhetorik in der postmodernen Welt. München: Trickster

Wolff, Stephan (1987): Rapport und Report. Über einige Probleme bei der Erstellung plausibler ethnographischer Texte, in: Werner von der Ohe (Hg.), Kulturanthropologie. Beiträge zum Neubeginn einer Disziplin, Berlin: Duncker \& Humblot, S. 333-364

\section{Unter Aborigines - Reflexionen über eine exotische Fremdheitserfahrung}

THOMAS S. EBERLE

Alfred Schütz hat Verstehensprozesse in all ihren Subtilitäten beschrieben. Zu seinen Grundeinsichten gehört, dass Fremdverstehen ein Transzendenzerlebnis ist: Das Bewusstsein des alter ego ist dem Deutenden nicht unmittelbar zugänglich; der subjektive Sinn, den der andere mit seinem Handeln verbindet, wird immer signitiv vermittelt. Aufgrund der unterschiedlichen biographiespezifischen Wissensvorräte mit ihren verschiedenen Relevanzstrukturen ist ein Verstehen selbst zwischen Mitgliedern derselben Kultur stets nur approximativ möglich. Um wie viel schwieriger müssen Verstehensprozesse sein, wenn die Beteiligten ganz unterschiedlichen Kulturen angehören?

Ich war schon öfters »in der Fremde« gewesen. Zunächst in den unmittelbaren Nachbarländern - Deutschland, Österreich, Italien und Frankreich -, dann in vielen anderen nord-, west-, süd- und osteuropäischen Ländern, schließlich in den Vereinigten Staaten, im Nahen Osten, in Indien und verschiedenen asiatischen Ländern. Ich war bei den HopiIndianern gewesen, hatte im Tipi den Zeremonien eines Schamanen beigewohnt, war tagelang mit Beduinen auf dem Kamel durch die arabische Wüste geritten und war im Okavango-Delta auf Safari. Doch nie hatte ich mich derart fremd gefühlt wie bei den Aborigines in Australien. Es war die maximale kulturelle Differenz, die ich bisher wam eigenen Leibe« erlebt hatte. 
Im Oktober 2000 war ich mit meiner Frau nach Australien gekommen, um hier mein Forschungssemester zu verbringen. ${ }^{1}$ Dabei war eine ganze Reihe von Feldaufenthalten bei den Aborigines geplant. Im Folgenden berichte ich über unsere allerersten Begegnungen, die noch durch maximale Unsicherheit und Ungewissheit gekennzeichnet waren. In einem ersten Teil beschreibe ich diese ersten Kontakte en bloc. Im Stil der lebensweltlichen Ethnographie (Honer 1993, 2000) skizziere ich meine Beobachtungen aus der subjektiven Erfahrungsperspektive. Im Gegensatz zu vielen klassischen Ethnographien und der Methode der teilnehmenden Beobachtung wird dabei das Beobachtete nicht vom beobachtenden Subjekt abgekoppelt, als ob es eine objektive Repräsentation wäre; vielmehr wird das Beobachtete konsequent aus der Perspektive des Beobachters beschrieben. Im zweiten Teil wechsle ich zur Perspektive der phänomenologischen Lebensweltanalyse (Hitzler/Eberle 2000) und stelle einige Reflexionen über die involvierten Verstehensprozesse an. Die Begegnungen mit den Aborigines waren eine wahrhaf exotische Fremdheitserfahrung, und zwar in beiderlei Wortsinne: »fremdländisch/überseeisch « als auch »aus dem Üblichen herausfallend « und »einen fremdländischen Zauber habend oder ausstrahlend « (Duden 1990: 238). ${ }^{2}$

\section{Unter Aborigines}

\section{Die ersten Kontakte in Darwin}

Die ersten Kontakte mit Aborigines hatten wir bereits kurz nach der Landung in Darwin, der Hauptstadt des Northern Territory im nördlichsten Teil Australiens. ${ }^{3}$ Darwin ist nicht nur die australische Stadt mit dem höchsten Bevölkerungsanteil an Aborigines, sondern auch ein idealer Ausgangspunkt, um jenes Gebiet zu erreichen, in dem noch am meisten Aborigines ihrer eigenen Lebensweise frönen: Arnhem Land. Wir hatten

1 Die folgenden Erlebnisse fanden am Anfang meines Forschungssemesters in Australien bei den ersten Feldaufenthalten in East Arnhem Land statt. Ich danke der Universität St. Gallen für die Unterstützung dieses Forschungsaufenthalts.

2 Der verbreitete Topos »der exotische Fremde« ist daher nicht wirklich ein Pleonasmus.

3 Australien besteht aus sechs Staaten und einigen »Territorien«, die früher selbständige Kolonien des Commonwealth bildeten und sich am 1.1.1901 $\mathrm{zu}$ einem Bundesstaat zusammenschlossen. Das Northern Territory hat dieselbe Organisationsform, aber nicht den Status eines selbständigen Staates. in Darwin noch einige Besorgungen zu machen, und das führte unweigerlich zu den ersten Begegnungen mit diesen dunkelhäutigen Menschen mit den ungewohnten Kopfformen. Es lassen sich retrospektiv drei deutlich konturierte Erfahrungstypen ausmachen: Zum einen trafen wir sie wohl integriert als Verkaufspersonal in Läden und Hotels, und viele von ihnen waren etwas hellhäutiger. Sie fielen uns nicht sonderlich auf und waren auch oft nicht eindeutig zu erkennen, gibt es doch viele Farbige hier - Darwin ist die multikulturellste Stadt Australiens, mit vielen neuen Einwanderern aus Süd- und Ostasien. Der zweite Erfahrungstyp ist einer der unangenehmeren Art: Wie alle Einheimischen und Touristen, trafen wir viele Aborigines vor allem auf der Strasse an, am Boden sitzend, bevorzugt am Eingang von Warenhäusern, und meist betrunken und bettelnd. Sie wirkten durchwegs verwahrlost, mit schmutzigen und zerrissenen Kleidern, vergilbten oder ausgefallenen Zähnen; es waren heruntergekommene Gestalten, die oft laut schrieen und aggressiv schienen. Dem Neuling, der die Situationen nicht richtig einzuschätzen vermag, jagen sie eher Angst ein, und die meisten machen daher kurzerhand einen Bogen um sie herum. Der dritte Erfahrungstyp schließlich: Man sieht sie im Kreis unter einem Baum im Park sitzen, stets im Schatten und weitgehend regungslos. Ob sie da meditieren? Oder was beraten? Oder einander einfach nur anstarren? Man weiß es nicht. Sie sitzen einfach da. Später werden uns einheimische Weiße herablassend sagen, dass Aborigines pro Tag nur drei Bewegungen ausführen: Am Morgen laufen sie zu ihrem Baum und setzen sich in dessen Schatten; am Mittag stehen sie auf und setzen sich auf die andere Seite des Baumes, um auch am Nachmittag im Schatten zu sitzen, und am Abend gehen sie wieder zurück zu ihrem Schlafplatz.

Als Fremder kann man die Situation nicht einschätzen: Sie sitzen in einem geschlossenen Kreis - man getraut sich nicht, einfach zu ihnen zu gehen und sich zu ihnen zu setzen, man würde da eindringen, schon rein von der Anordnung der Körper her wäre man ein Eindringling, dann aber auch von der Hautfarbe her, von der Kultur her - ja, in jedem Sinne. Eine Erinnerung bleibt jedoch haften: Als wir in dem Touristentram eine Sight-Seeing-Tour durch die Stadt unternahmen, winkte uns eine Aborigine-Frau, die im Kreis mit anderen im Schatten eines Parkbaums $\mathrm{saß}, \mathrm{zu}$ : Es war eindeutig ein Winken und wirkte freundlich, ich vermutete denn auch gleich ein Lächeln, doch war das nicht wirklich zu erkennen. Dieses Winken freute mich, es wirkte fröhlich-vergnügt, mein Herz wurde warm - war es vielleicht mehr als nur ein Gruß? War es gar eine Einladung, mit ihr in näheren Kontakt zu treten? Sich zum Kreis dazuzugesellen? Oder würden sie uns dann nur anbetteln oder gar belästigen? $\mathrm{Zu}$ spät, das Tram fuhr weiter und hielt erst viel später an der nächsten 
Station. Es war dies der erste direkte Kontakt mit »wirklichen« Aborigines, einprägsam zwar, doch nur sehr flüchtig, vielleicht eine verpasste Gelegenheit?

\section{Ankunft in East Arnhem Land}

Wie dem auch sei - wir hatten Besseres vor: Wir wollten zu jenen $\mathrm{Ab}$ origines, die nicht in den Städten der Weißen, sondern weit abgeschieden auf ihrem eigenen Territorium lebten: zu den Aborigines in Arnhem Land. Arnhem Land ist ein Siedlungsgebiet der Aborigines, das mi $97000 \mathrm{~km}^{2}$ mehr als doppelt so groß ist wie die Schweiz, aber weitgehend dürr und unfruchtbar. Mit seinen 20.000 Menschen ist es insgesam spärlich besiedelt, enthält aber eine der größten Aborigines-Populationen Australiens. 1976 hat die australische Regierung die Aborigines als rechtmäßige Eigentümer anerkannt. Das gesamte Gebiet ist für Fremde gesperrt; wenn man es betreten will, braucht man eine Bewilligung, um die man am besten lange im voraus nachsucht.

So flogen wir also in einem kleinen Flugzeug nach Gove, einer kleinen Siedlung am äußersten Nordzipfel der Gove Peninsula, wo es eine berühmte Bauxit-Mine und eine Aluminium-Raffinerie gibt. Noch vor wenigen Jahren konnten sich die hier lebenden Weißen frei bewegen, doch seit einiger Zeit war nun jeglicher Zugang zum Territorium der Aborigines für Fremde gesperrt. Als Fremde gelten nicht nur Touristen, sondern auch sämtliche Australier, die keine Aborigines sind. Der Feldzugang war daher außerordentlich schwierig. Wir hatten eine kleine Expedition zu zweit mit einem lokalen Guide gebucht, der uns zu den $\mathrm{Ab}$ origines führen und uns den Zugang ermöglichen (»die Türen öffnen《) sollte. Aufgrund einer Fehlbuchung der Agentur war dieser aber für längere Zeit ortsabwesend. Glücklicherweise logierten wir in einem Motel, dessen Besitzerfamilie selbst freundschaftliche Beziehungen $\mathrm{zu}$ den Aborigines unterhielt. Sie vermittelte uns den Kontakt mit einer befreundeten Aborigine-Frau, die bei ihrem Stamm als Lehrerin wirkte und, als eine der wenigen, über Englisch-Kenntnisse verfügte. Wir trafen uns beim lokalen Aboriginal Art Centre, das von Fremden noch ohne spezielle Bewilligung mit dem Auto angesteuert werden durfte.

Schon die Begrüßung verlief ziemlich ungewohnt: Wir hatten die Lehrerin wie verabredet angetroffen, sie stand da mit einer anderen Frau, aber wir fühlten uns weder erwartet noch willkommen: Beide würdigten uns Fremde mit keinem Blick, schlenderten gemächlichen Schritts an uns vorbei, als ob wir Luft wären, und sprachen nur mit unserer Motel-Besitzerin, die uns hergebracht hatte. Erst als diese uns mitei- nander förmlich bekannt machte, drückten sie uns flüchtig die Hand und murmelten etwas Unverständliches, aber ohne uns anzusehen. Sie wirkten beide nicht sehr motiviert, vielmehr schienen sie sich aus irgendeinem Grund dem Wunsch unserer Motel-Besitzerin zu fügen. Allerdings hatten sie uns bereits einen >Permit火 verschafft und waren bereit, uns zu begleiten. ${ }^{4} \mathrm{Mal}$ sehen, wie das herauskommen würde... Der Anfang verlief denn auch nicht sehr vielversprechend: Obwohl die Motel-Besitzerin der Gundumuk ${ }^{5}$ eindringlich erklärt hatte, dass wir im Gegensatz zu den (wenigen) anderen weißen Touristen nicht an der Kunst, sondern an der Lebensweise der Aborigines interessiert seien, führte sie uns direkt ins Art Centre. Zunächst fühlten wir uns darob etwas düpiert; da die Ausstellung jedoch wirklich beeindruckend war, ließen wir uns darauf ein. Einem Ausstellungsprospekt entnahmen wir, dass im Arnhem-Land weltberühmte Künstler leben und hier auch ausstellen. Persönlich waren wir von vielem sehr angetan, und schließlich kauften wir - nach rein ästhetischen Gesichtspunkten - einen großen »Hollow-Log $«{ }^{6}$ den wir uns nach Hause schicken ließen. Beim Kaufabschluss stellte sich heraus, dass er von einer sehr bekannten Künstlerin bemalt worden war, von der auch Kunstwerke in der National Gallery in Canberra stehen.

Unsere Freude an der Ausstellung wurde von unseren zwei Begleiterinnen sehr wohl registriert, und es schien mir, also ob sie dadurch etwas auftauten. Hatten sie sich zunächst sehr im Hintergrund gehalten, teilweise wirklich unsichtbar, kamen sie nun näher und traten sogar mit uns in Kontakt: Gundumuk zeigte uns in einer der aufgelegten Mappen einige Zeichnungen, die sie selbst gefertigt hatte, und dasselbe tat ihre Begleiterin. Das waren nun allerdings alles andere als Kunstwerke, sie sahen aus wie Kinderzeichnungen oder Übungsskizzen, womöglich aus einem Zeichenkurs für Anfänger. Trotzdem lobten wir die Zeichnungen, nicht aus Freude an deren Qualität, aber aus Freude daran, dass sie sich selbst auch in Kunst übten und uns ihre Werke zeigten. Gleichzeitig stellte sich eine Unsicherheit darüber ein, ob sie unsere Freude nun an

4 Selbst mit einem Permit darf man sich auf Aborigines-Gebiet nicht frei bewegen, sondern muss die ganze Zeit in Begleitung einer einheimischen Person sein.

5 Name geändert.

6 Hollow-Logs sind von Termiten ausgehöhlte Eucalyptus-Stämme, die aber wesentlich größer sind als Didgeridoos. Sie sind außen kunstvoll verziert
mit den Totems des jeweiligen Clans. Ihre besondere Funktion erhalten sie mit den Totems des jeweiligen Clans. Thre besondere Funktion erhalten sie in der Dupun-Zeremonie, während der die Knochen eines verwesten ClanMitglieds bemalt und in die Öffnung eingefüllt werden. Mit Tänzen soll
sichergestellt werden, dass die Seele des Verstorbenen sicher im Land der Toten ankommt. Anschließend verwittert der Knochensarg unter dem Einfluss der Naturelemente. 
ökonomischen Indikatoren messen würden: Hegten die beiden nun woh die normative Erwartung, dass wir als Zeichen unserer Dankbarkeit für ihre Gastfreundschaft - dafür dass sie uns begleiteten - oder als Zeichen der Echtheit unserer Freude eine ihrer Zeichnungen kaufen? Da das Art Centre nur als Agent handelt, würden sie selbst direkt davon profitieren. Erinnerungen an andere Länder stellten sich ein: Im Orient, in arabischen Ländern oder in Indien, überall hatte man uns gastfreundlich empfangen - um gleich darauf aber auch unmissverständlich kund zu tun, dass wir den Gastgebern etwas abkaufen sollten. War das hierzulande ähnlich? Sollten wir nun etwas kaufen, das wir gar nicht wollten - nur um jemandem eine Freude zu bereiten?

In solchen Situationen kultureller Ungewissheit, in denen man selbst unmittelbar vor einer Handlungsentscheidung steht, stellt sich der Wunsch ein, jemanden fragen zu können, der in der Lage ist, einem kompetent Auskunft zu geben. Mein Blick schweifte zu den beiden Weißen, die - wie sich gleich herausstellte - die Manager des Art Centres waren. Selbst hier, mitten in Arnhem Land, waren die Leitungspersonen Weiße. Sogleich verband ich damit die Vermutung, dass sie kulturell etwa gleich tickten wie wir, dass ich sie also sicherlich diskret fragen dürfe, was in Bezug auf mein Handlungsproblem hierzulande normadäquates Verhalten sei. Aus der Tatsache, dass sie hier lebten und das Aboriginal Art Centre leiteten, schloss ich zudem auf ihre Vertrautheit mit den Sitten und Bräuchen der hiesigen Eingeborenenstämme. In der Tat war der eine, wie sich später herausstellte, auch mit einer AborigineFrau verheiratet. Es bestünden keinerlei Kauferwartungen von Seiten unserer Begleiterinnen, wurde uns beschieden, es reiche ihnen vollauf, wenn wir ihnen unsere Freude bekundeten. Die innere Spannung, die sich aufgebaut hatte, löste sich auf in Erleichterung: Dann konnten wir uns also ganz normal benehmen und würden sie weder enttäuschen noch beleidigen.

\section{Beim Fischen am Meer}

Wir verließen das Art Centre und fuhren zu viert in unserem Jeep in den Wald. Gundumuk wies uns den Weg - wir wussten nicht, wohin die Reise ging. Plötzlich öffnete sich der Wald und gab den Blick frei auf eine wunderschöne Bucht, gesäumt von einem weißen Sandstrand, der gegen den Wald anstieg und mit Felsbrocken unterschiedlichster Größe durchsetzt war. Wir hielten an und bestaunten die wunderschöne Szenerie. Gundumuk gab uns zu verstehen, dass wir hier picknicken sollten. So packten wir die mitgebrachte Kühlbox aus, und es war ganz klar, dass das für drei Personen vorgesehene Essen nun auf vier Personen verteilt wurde - es war auch mehr als genug da. Achtzig Meter weiter saß und lag eine größere Anzahl Aborigines verstreut (also nicht im Kreis) unter den Bäumen, bunt gemischt: Frauen und Männer ganz unterschiedlichen Alters, viele Kinder, aber auch einige Greise. Es waren insgesamt etwa zwei Dutzend Personen, im Laufe des Nachmittags stießen dann noch weitere dazu. Die Begleiterin von Gundumuk spazierte sogleich rüber, um sie zu begrüßen, und wir beobachteten, dass sie ihren Anteil am Essen teilweise weitergab.

Erst später erfuhren wir, dass es eine Grundregel unter Aborigines ist, Nahrung zu teilen. Im Nachhinein ist mir nicht klar, warum wir im Abstand von den anderen, also in Distanz und ganz für uns gegessen hatten - vielleicht, weil sonst für uns nichts mehr übrig geblieben wäre? Denn kaum waren wir fertig, schlug Gundumuk vor, dass wir zu diesen Leuten hinübergehen. Wir nahmen das Angebot gerne an, begrüßten die Fremden mit winkenden Hand- und Armbewegungen, und wir fühlten uns sofort willkommen. Die meisten lachten freundlich, mehrere winkten uns näher, und so setzten wir uns mitten unter sie. Unsere Begleiterinnen ließen sich einige Meter entfernt von uns nieder, was uns etwas komisch anmutete: Sie fühlten sich offenbar nicht verpflichtet, uns vorzustellen oder als Übersetzerinnen zu walten. So begann nun das große Kommunikationsproblem: die meisten verstanden kaum Englisch, höchstens einige Brocken. Einfachste Sätze stellten jeweils einen Kurzkontakt zwischen uns her, ließen die verbale Kommunikation aber auch sogleich wieder zusammenbrechen. Die Begleiterin von Gundumuk wiederholte mehrmals und in bedächtigem, fast beschwörendem Tonfall: $\gg$ Aborigines good people! «, und dann: »Aborigines nice people, Aborigines very nice people $\ll$.

So hockten wir also da und fühlten uns ziemlich ausgestellt, da wir von allen Seiten - versteckt zwar, doch spürbar - beäugt wurden. Munter plauderten die Leute in ihrer eigenen, für uns völlig unverständlichen Sprache weiter. Es fiel uns auf, wie oft sie herzhaft lachten - man konnte nur spekulieren, worüber. Möglicherweise lachten sie über uns. Das wurde etwa manifest, als wir etwas über ihre Kinder sagten. Diese spielten nackt an einer Wasserquelle an der Straße, wo sie sich ausgelassen gegenseitig bespritzten, und es bot sich uns ein traumhaft schönes Bild, als die Sonnenstrahlen durch die Zweige und Blätter der Bäume drangen und ein vielschichtiges Spiel von Licht und Schatten hervorzauberten. Die Kinder waren völlig schwarzhäutig, spielten ganz verzückt und schauten äußerst anmutig aus. So sagten wir entzückt: »You have beautiful children!« Sogleich ging ein Raunen durch die Erwachsenengruppe und jemand wiederholte laut und in einem spöttischen oder amüsierten 
Klang: »beautiful children! - und irgendwie war mir von der Art ihrer Reaktion her klar, dass sie unsere Aussage kommentierten im Sinne: Die Kinder findet ihr hübsch - doch was ist mit uns? Sofort war mir klar Mit jeder Aussage kann man fortan in ein weiteres Fettnäpfchen treten.

So taten wir es den Aborigines gleich: Wir saßen einfach da. Gundumuk erklärte uns später, dass diese (Groß-)Familie jeden Tag hierher komme. Jeden Tag also, stellte ich mir vor, setzten sie sich in den Schat ten dieser Bäume und blickten aufs Meer hinaus. Alle sahen sehr entspannt aus. Schon binnen kurzem realisierten wir, dass hier die Zeit völlig anders tickt: Es herrscht Ruhe, Gemächlichkeit und Gelassenheit Stunden erschienen mir wie Tage, und die Atmosphäre wirkte sehr erholsam. Zugleich fiel mir der hohe Wachheits- und Aufmerksamkeitsgrad auf, den diese Leute pflegten: Leiseste Töne oder die kleinste Bewegung eines Vogels nahmen sie sofort wahr, selbst wenn dieser mehrere hundert Meter von uns entfernt war. Sie konnten mit plötzlicher Aufgeregtheit auf irgendein Tier hinweisen, das ich nur mit größter Mühe oder meist gar nicht erkennen konnte. Auf dem Hintergrund dessen, das hier so wenig passiert, wurde jede Bewegung aufmerksam registriert. Allerdings hatte dies einen weiteren Grund, den wir sogleich erkennen sollten: Die Leute, die hier so gemütlich herumsaßen, waren in Wirklichkeit auf der Jagd.

Etwas abseits, auf einem größeren Felsbrocken, saß ein älterer Mann mit weißem Haar und ergrautem Bart: eine würdevolle, prächtige Erscheinung. Die ganze Zeit hatte ich ihn immer wieder bewundert - er war ein Anblick wie im Bilderbuch. Nur zu gern hätte ich ihn - wie zuvor schon die Kinder - fotografiert. Doch soviel wussten wir aus dem Reiseführer: Aborigines darf man nicht fotografieren, denn sie glauben an die Magie der Bilder - durch den Anblick von Fotos beeinflusst man die Seele des abgebildeten Menschen. Manchmal geben Sie einem aber trotzdem die Erlaubnis, ein Foto zu machen, wenn man sie fragt. Es schien mir nun aber ziemlich unsensibel, so kurz nach unserer Ankunft mit der Kamera aufzufahren und sie um Fotoaufnahmen zu bitten. Dieser altehrwürdige Mann hatte längere Zeit einfach da gesessen und aufs Meer hinaus geschaut und sich an der Kommunikation nicht beteiligt. $\mathrm{Ja}$, er schien überhaupt keine Notiz von uns Fremden genommen zu haben. Doch nun stand er mit einem Ruck plötzlich auf, ergriff drei neben ihm liegende Speere und ging zum Strand hinunter. Sofort ging ein Raunen durch die Menge, Kinder und Jugendliche stoppten ihr Spiel und aller Augen richteten sich auf das, was sich nun abspielen würde. Allen war klar, worum es ging, nur uns Fremden nicht: Er hatte in der Bucht einen Kingfish gesichtet, erklärte uns Gundumuk. Alle sahen diesen, nur wir nicht. Unseren Augen bot sich lediglich ein Farbenspektakel: das
Weiß des Sandes und dahinter die verschiedenen Blautöne des Meeres von Türkisfarben bis Tiefblau. Der Alte schritt den Strand entlang hin zur rechten Seite der Bucht, und in ca. 100 Meter Entfernung von uns begann er mit seinen langen, dürren Beinen langsam, vorsichtig und mit großen Schritten durchs Wasser zu waten. Mit ruhiger Hand setzte er einen Speer zum Wurf an, zielte und schleuderte ihn mit Wucht ins Wasser. Der Speer blieb in etwas stecken, das langsam weiter schwamm, in Richtung des tieferen Wassers. Der Alte folgte dem verwundeten Tier und setzte einen zweiten Speer zum Wurf an. Dieser wirkte offenbar tödlich, denn nun blieb der getroffene Fisch reglos im Wasser liegen. Der Alte stieß ihn mit den Füßen ans Ufer und ließ ihn dort liegen. Ruhigen Schritts ging er wieder zu seinem Felsen zurück, setzte sich und blickte wieder aufs Meer hinaus. Er hatte keinen Laut von sich gegeben. ${ }^{7}$

Nun kam unter den jungen Männern Betriebsamkeit auf: Die einen holten den ca. $120 \mathrm{~cm}$ großen Fisch vom Strand, die anderen entfachten ein Feuer, die dritten holten würzige Blätter aus dem Wald, und die vierten nahmen den Fisch aus, wobei die Leber als besondere Delikatesse galt und einem eigenen Kochprozess zugeführt wurde. Es herrschte eine offenkundig bestens eingespielte Arbeitsteilung, jeder wurde aus eigenem Antrieb tätig, oft von einer Schar Kinder begleitet, die sich das besondere Ereignis des Tages nicht entgehen lassen wollten. Der Fisch garte nun auf verkohltem Holz in einem Sandloch unter einem rostigen Blech, das die Hitze konservierte. Später wurde der Fisch in Stücke geschnitten und unter sämtlichen Anwesenden verteilt. Wir konnten nicht erkennen, ob der Verteilungsprozess irgendeiner Systematik folgte. Als wir nach dem Essen rauchten und unseren Gastgebern Zigaretten offerierten, bedienten sich die meisten Erwachsenen, und zwar oft gleich mit zwei, und nun konnten wir gut beobachten, wie die Zigaretten innert kürzester Zeit ihre Besitzer wechselten. So gab es anschließend einzelne, die viele Zigaretten hatten, und andere, die leer ausgingen. Auch hier blieb uns die Logik des Verteilungsprozederes verborgen; vermutlich herrschte ein komplexes Geflecht von Tauschbeziehungen - eine Zigarette abgeben beglich eine vergangene Schuld oder kreierte künftige Debitoren.

7 Erst später wurde uns bewusst, wie einzigartig dieses Erlebnis war: Erstens gibt es nur sehr wenige Australier, die je Zeuge einer solchen Fischjagd waren, und zweitens haben uns weiße Fischer versichert, dass es praktisch aussichtslos sei, mit einem Speer einen Fisch erlegen zu wollen. Keinem von denen, die es versucht hatten, war es je gelungen. Der Speerwurf muss töđlich sein - und das erfordert höchste Präzision -, sonst schwimmt der Fisch mit dem Speer im Leib davon. 


\section{Im Dorf}

Obwohl auch diese Aborigines mittlerweile in Häusern und nicht meh in Hütten aus Palmblättern wohnen, leben sie noch großenteils wie ihre Vorfahren: Sie leben nach wie vor als Großfamilien auf engstem Raum und jeden Tag gewinnen sie der Natur nur soviel ab, wie sie zum Essen brauchen. Dazwischen sitzen oder kauern sie auf dem Boden, schauen stundenlang aufs Meer hinaus, registrieren jede auch noch so kleine Bewegung in der Natur, und dazwischen reden sie miteinander. Materialismus ist diesen Leuten fremd: Sie töten nicht mehr Fische, als sie heute essen können, und legen keine Vorräte an; sie haben kein Interesse, einen Job in der Wirtschaft auszuüben, um auf dem landesüblichen Niveau wirtschaftlichen Wohlstands leben und sich die gängigen Konsumgüter leisten zu können, und sie interessieren sich weder für Wachstum noch für persönliches Eigentum.

Gegen Abend fuhren wir mit Gundumuk ins Dorf, wo sie uns zu zwei verwandten Familien (aber nicht zu ihrer eigenen) brachte, erst zur einen, dann zur anderen. Das Dorf bestand aus einer einfachen Baracken-Siedlung mit Wasserhähnen entlang der ungeteerten Straße, wahrscheinlich alles von der Regierung gesponsert. Bei beiden besuchten Familien saßen mehrere Frauen auf dem Boden der Veranda vor dem Hauseingang und machten Kunst: Die eine bemalte ein Didgeridoo, die andere einen Bumerang, und eine Alte flocht etwas aus getrocknetem Gras. Sie wirkten alle sehr schüchtern, fast scheu. Es schien sie indes nicht zu stören, wenn wir hinzu traten und ihr Tun aus der Nähe betrachteten, vielmehr hatten wir den Eindruck, dass sie unser positives Feedback jeweils freute und ein Lächeln über ihr Gesicht huschen ließ. Einen besonderen Anknüpfungspunkt für die Kommunikation boten die Kleinkinder in den Armen von Frauen: Sogleich fand sich meine Frau in einem intensiven interaktiven Austausch, der sich allerdings völlig nonverbal, allein über Mimik und Gestik vollzog: das Baby betrachten, freudig lachen, mit ihm Blickkontakt herstellen, seine Ärmchen und Beinchen ergreifen und hin und her bewegen, es streicheln, es in die Arme nehmen und wiegen, Freude über seine Reaktionen zum Ausdruck bringen usw. - und all das immer interaktiv abgestimmt mit der Mutter bzw. der zuständigen Frau. So konnte in diesen Begegnungen auch ohne Worte ein Gefühl von Verbundenheit hergestellt werden: die gemeinsame Freude am Kind.

\section{Beim zweiten Stamm}

Gundumuk begleitete uns zurück zum Aboriginal Art Centre, wo wir uns bedankten und von ihr verabschiedeten. Die Nacht verbrachten wir im Motel, und am nächsten Tag wollten wir einen weiteren Stamm besuchen. Die Tochter der Motel-Besitzer - die Managerin des Hauses hatte sich von einer Aborigines-Familie adoptieren lassen. Dies ist be den hiesigen Aborigines ein verbreitetes Verfahren: Weiße können (auch als Erwachsene) adoptiert werden, wodurch sie in die Familie aufgenommen werden. Ganz allgemein ist die Blutsverwandtschaft bei den Aborigines weit weniger wichtig als die soziale und insbesondere die kulturelle Zugehörigkeit. ${ }^{8}$ Adoptiert zu sein heißt nun aber nicht, das man jederzeit Zutritt zu ihrem Territorium hat: Man muss immer zuerst die Zustimmung des Adoptivvaters einholen, wenn man die Familie be suchen will. Mary ${ }^{9}$ wollte uns unbedingt »ihre« Familie vorstellen, zumal »ihr« Vater als einer der besten Didgeridoo-Spieler Australiens galt Doch ausgerechnet jetzt war die Zeit ungünstig: Vor wenigen Tagen hatte ein junger Verwandter seine Tante umgebracht, und jetzt herrschte große Unruhe im Stamm. Verwandte von überall her waren angereist um mit $\mathrm{zu}$ beraten und zu debattieren, mögliche Retorsionsmaßnahmen und Gegenleistungen wurden erörtert, und es war offenbar viel Aggression in der Luft. Die Polizei hatte den Mörder zwar verhaftet und in ein Gefängnis im 1000 Meilen weit entfernten Alice Springs überführt, um die Situation vor Ort zu entschärfen. Doch solche Fälle werden bei den Aborigines nach eigenen Gesetzen beurteilt und geahndet, unabhängig vom australischen Staat - und oft in Konflikt mit ihm. Im vorliegenden Fall, so erklärte uns der Motel-Besitzer, würde dem Täter mindestens mit einem Speer der Oberschenkel durchbohrt - für die staatliche Rechtsprechung ein weiteres Delikt.

Noch vorige Nacht war eine wilde Schlägerei ausgebrochen, bei der es mehrere Verletzte gab, und so riet uns der Adoptivvater von einem Besuch ab. Am Mittag hieß es dann überraschend, wir könnten nun doch kommen. Mary begleitete uns und fuhr uns hin. Wieder trafen wir eine Siedlung mit einfachen Holzhäusern an, doch war sie direkt am Meer

$8 \mathrm{Zu}$ heißen Diskussionen führt dies in der australischen Politik, wenn es um die Anerkennung der Stammeszugehörigkeit konkreter Personen und damit ihres Eigentumsanspruchs auf ein Territorium geht, das den Aborigines zugesprochen wurde. So behaupten beispielsweise die einen, der letzte
Tasmanier sei 1876 gestorben, während sich heutzutage eine Gruppe als Tasmanier sei 1876 gestorben, während sich heutzutage eine Gruppe als erhalten und die angestammte Lebensweise pflegen. Viele von ihnen sind hellhäutig.

9 Die Namen sind geändert. 
gelegen. Das Haus von Marys Adoptivfamilie stand an vorderster Front an einem breiten und langen Sandstrand, der gemeinsam mit Felsen in einem weitem Bogen wiederum eine größere Bucht formte. Die älteren Frauen der Familie saßen auf Decken im Sand, die Kinder tollten umher, und auch die jüngeren Leute waren in Bewegung. Auf der Veranda saBen vier ergraute Männer nebeneinander auf Stühlen und blickten eher grimmig drein, zumindest schlecht gelaunt oder besorgt oder nachdenklich. Sie wirkten wie in sich gekehrt und nahmen keinerlei Notiz von uns. Auch die anderen behandelten uns Fremde wieder wie Luft, nur Mary kam in Kontakt mit ihnen.

Die Situation war für uns wesentlich anders als gestern: Mary hatte uns auf der Hinfahrt bereits viele Fragen beantwortet und etliche $\mathrm{Ge}$ schichten erzählt, und diese rahmten nun unsere Begegnung und luden unsere Interpretationen mit zusätzlicher Bedeutung auf. Wir hatte Kenntnis von dem Mord, der Schlägerei, den vielen Verwandten, die si als Gäste zu betreuen hatten - entsprechend sahen wir »grimmige« Gesichter, »schlecht gelaunte« Gestalten, »besorgte« oder »nachdenkliche« Blicke. Wir spürten förmlich, dass etwas Schweres auf dieser Gemeinschaft lastete und die Stimmung drückte. Wir wussten auch, dass dies ein christianisiertes Dorf war, was die Sache für uns insofern besonders interessant machte, als die kollektive Verarbeitung des Mordes nun mi dem Wertekonflikt zwischen den christlichen Geboten der Nächstenliebe, Barmherzigkeit und der Vergebung einerseits und den alten Stammestraditionen andererseits zurande kommen musste. Des Weiteren hatten wir auch erfahren, dass dies ein so genanntes »trockenes « Dorf war, d. h. es herrschte totales Alkoholverbot. Dies ist nicht unbedeutend, da nicht nur viele Aborigines in den Städten, sondern ganze Aboriginal Communities unter der Alkoholsucht leiden.

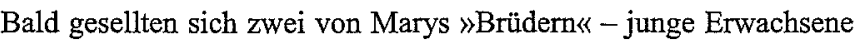
- zu uns. Beide sprachen etwas Englisch, und so waren sie auch die primären Ansprechpartner für Mary (die sich nicht bemühte, die Sprache der Eingeborenen zu erlernen). Da auch diese beiden uns keines Blickes würdigten, stellten wir unsere Fragen an Mary, die sie - weil sie viele von ihnen selbst nicht beantworten konnte - sogleich an ihren älteren "Bruder« richtete. Die Antworten kamen oft etwas zögernd daher, durchsetzt mit Pausen, und blieben oft vage. Als konversationsanalytisch geschulter Beobachter fragte ich mich unwillkürlich, was das wohl zu bedeuten habe. Sein Englisch schien mir jedenfalls kaum auszureichen, um unsere Fragen überhaupt »richtig « zu verstehen und stimmig beantworten zu können. Die unkritische Art, wie Mary seine Antworten kolportierte, mahnte uns jedenfalls zur Vorsicht gegenüber dem Wahr- heitsgehalt ihrer Geschichten und ihrer Antworten auf unsere Fragen. Da war wohl manches nicht zum Nennwert zu nehmen.

Da die Jungen - von den Kindern ganz zu schweigen - rasch wieder ihren eigenen Aktivitäten nachgingen, blieben wir längere Zeit bei den Alten sitzen, vorwiegend Frauen, aber auch einem sehr alten Greis (der offenbar nicht mehr zum Ältestenrat gehörte, der auf der Veranda tagte). Wieder schienen sie dumpf vor sich hinzustarren, sie hoben nie ihr leicht gesenktes Haupt, schauten uns nie an und blickten uns nie in die Augen, auch gaben sie keinen Laut von sich. Meine Frau, die mit ihrem langen, blond gelockten Haar für die Aborigines immer wieder die Hauptattraktion darstellte, bemerkte aber, wie intensiv sie gemustert wurde: Ganz versteckt, mit kaum wahrnehmbaren Blicken schielten die Alten aus ihren tiefen Augenhöhlen hervor und zwischen ihren langen Haarsträhnen hindurch. Später erfuhren wir, dass Aborigines eben sehr scheu und schüchtern seien und Weiße nicht direkt anzublicken, geschweige denn anzusprechen wagten. Das machte es in dieser Situation sehr schwierig, mit diesen Alten in Kontakt zu kommen, denn da sie kein Englisch beherrschten, blieb nur die non-verbale Kommunikation, und diese funktioniert (zumindest nach westlichem Verständnis) als Interaktion nur, wenn man einander anschaut.

Mary, die vorübergehend mit ihren $»$ Geschwistern« am Strand unterwegs gewesen war, kam nun zurück und lud meine Frau ein, mit ihr und den andern Frauen Muscheln sammeln zu gehen. Kurz darauf tummelte sich eine Gruppe von neun jungen Frauen im seichten Wasser. Alle waren sie in den Kleidern ins Wasser gegangen - eine Folge der Christianisierung -, und nun waren nur noch ihre Köpfe sichtbar, die sich nur langsam bewegten und gleichsam über der Wasseroberfläche zu schweben schienen. Es herrschte ein munteres Geplauder, und es wurde auch viel gelacht. Nachher erklärten sie mir, dass sie am Boden gekauert sind und mit beiden Händen den Sandboden durchwühlt haben, um $\mathrm{Mu}$ scheln zu finden. Den Rock zur Trageschürze geformt, trugen sie nach ungefähr einer Stunde ihre Beute an Land - für das Abendessen war gesorgt. Mary erzählte uns, dass dies ein typischer Frauen-Anlass sei, bei dem wacker geklatscht und getratscht wird und auch die Männer durchgehechelt werden.

\section{Regelverstöße}

Später signalisierten fünf der jungen Männer Aufbruchstimmung, und Mary lud uns ein, gemeinsam mit ihnen im Jeep zur nächsten Bucht zu fahren. Auf dem Weg erklärte sie uns den »tieferen« Grund: Die Jungs 
hatten Hunger. Aufgrund der vielen Verwandten, die in den letzten Tagen ernährt werden mussten, war fast nichts übrig geblieben. Also beschlossen die Söhne des Hauses in Absprache mit der Mutter, dass sie das verbliebene Brot als Proviant mitnehmen dürfen. Hätten sie das in ihrer Hütte ausgepackt, so wären sie verpflichtet gewesen, es auch allen anderen zu offerieren und mit ihnen zu teilen. Mit der Fahrt zur nächsten Bucht konnten sie die Norm, dass Nahrung gemeinschaftlich geteilt wird, umgehen. Diese Regelumgehung war für uns unmittelbar nachvollziehbar: Essen ist ein Grundbedürfnis, und wenn man an Hunger leidet, versucht man ihn zu stillen - wenn nötig auch unter Umgehung gewisser Verhaltensvorschriften. Der ganze Ausflug machte allerdings überhaupt nicht den Anschein, dass es ums Essen gehen könnte. Zunächst gab es zahlreiche Stopps, an denen der älteste Bruder ausstieg und einige dünne Eucalyptus-Stämme inspizierte. Seine Handlungen sahen nach Betasten, Befühlen und Überprüfen aus. Erst beim dritten Stopp realisierten wir, worum es ging: Da kam er nämalich zum Jeep zurück und holte eine Axt, und kurz darauf fällte er mit wenigen Hieben einen kleinen Baum. Er schlug ein ca. $120 \mathrm{~cm}$ langes Stück heraus, hob es an den Mund und blies in das von Termiten ausgehöhlte Loch hinein: Ein dumpfer, anhaltender Klang ertönte - ein neues Didgeridoo war geboren. Was mich dabei überraschte: Der junge Aborigine bewegte sich hier im Wald, als ob er zum ersten Mal da wäre und jede Minute neue Entdeckungen machen könnte. Obwohl er sich nie weiter als dreißig Meter von unserer Strasse weg bewegte, schien er die potentiell geeigneten Bäume nicht schon früher ausgekundschaftet und inventarisiert zu haben. Er schien keinen Plan im Kopf zu haben, sondern schritt anscheinend völlig spontan und eher zufällig auf gewisse Bäume zu, so als ob er sie noch nie gesehen hätte. So dauerte es eine ganze Weile, bis wir am Strand der nächsten Bucht ankamen, wo alle ein wenig ausschwärmten. Fast unmerklich vertilgten die Jungs nun ihre Brote, etwas abseits, durch Büsche verdeckt, unseren Blicken weitestgehend entzogen - wollten sie ihr Essen auch vor uns verbergen?

Unsere Aufmerksamkeit war mittlerweile auf Hunderte von leeren Bierflaschen gerichtet, die schön ordentlich auf einen Haufen geschichtet waren, gut einen Meter hoch und zwei Meter breit. Hierhin zogen sie sich also zurück, wenn sie Bier trinken wollten! Die strockene Stadt« galt offenkundig nur im Dorf, nicht aber in der nächsten Bucht. Hier also wurde ausgelebt, was dort verboten war! Den Soziologen überraschen solche Abweichungen nicht - wo es Normen gibt, erwartet er auch Devianz. Wir konnten indes nicht eruieren, welches die kollektiven Praktiken des Biertrinkens waren: Findet es regelmäßig statt oder nur zu bestimmten Anlässen? In welchem Rhythmus werden diese Flaschenberge abgeräumt? Sind sie das Resultat jahrelangen Biertrinkens oder lediglich einer einzigen Party? Gibt es noch andere Orte, wo getrunken wird? Sind daran nur die Männer beteiligt oder auch die Frauen? Nur die Jungen oder auch die Alten? Trinken sie einfach ein, zwei Bier oder besaufen sie sich bis zum Exzess? Nun, auf den Bierkonsum wollten unsere eingeborenen Begleiter partout nicht näher $\mathrm{zu}$ sprechen kommen - möglicherweise wegen der Gegenwart Marys, die als überzeugte Christin kirchlich engagiert war und das Verbot des Alkoholkonsums sinnvoll und wichtig fand. Wir spürten anhand ihrer ausweichenden Reaktionen, dass dieses Thema tabu bleiben soll, und so drangen wir nicht weiter in sie ein.

Interessant war umgekehrt die Beobachtung, wie normenkonformes Verhalten der Aborigines westlich geprägte Menschen irritieren kann. Schon als wir tags zuvor am Strand die Zigaretten verteilt hatten, staunten wir, wie rasch sie sehr ungleich umverteilt waren. Mary passierte heute etwas Ähnliches: Da sie davon wusste, dass die Nahrungsmittel alle waren, brachte sie ihrem Adoptivvater einen großen Fisch mit, den ihr leiblicher Vater persönlich gefangen und tiefgefroren hatte. Doch kaum hatte sie den Fisch übergeben, landete er bereits bei einem anderen. Obwohl diese Praktik des Weitergebens mit dem christlichen Gebot der Nächstenliebe kompatibel sein müsste, litt Mary sichtlich darunter. Sie fand es einfach "nicht recht« (not right), dass ihr Adoptivvater und seine engere Familie nun wieder nichts zu essen hatten. Der (eigenhändig gefangene) Fisch war gemäß dem westlichen Muster des Gebens und Nehmens als persönliches Geschenk intendiert, und so empfand sie das sofortige Weitergeben desselben ein wenig als Affront bzw. die hiesigen Regeln als »ungerecht«. Am Abend bat sie denn ihren (leiblichen) Vater, ihr nochmals einen Fisch herauszurücken, damit ihr Adoptivvater doch noch zu seinem Fisch komme. Ihr Vater war allerdings alles andere als begeistert von dieser Idee: Seine Tochter, klagte er uns später, sei einfach zu gutgläubig und merke nicht, wie sie von den Abos ausgenutzt werde.

\section{Analysen und Reflexionen}

Der vorstehende Erfahrungsbericht unserer ersten Begegnungen mit Aborigines bietet ausreichend Material, um mannigfaltige Arten von Analysen durchzuführen. Auch die einzelnen Sinndeutungsprozesse könnten bis in mikroskopische Details hinein durchleuchtet werden. Im Folgenden beschränke ich mich darauf, einige Grundzüge des Verstehens dieser exotischen Fremdheitserfahrung herauszuarbeiten. In theore- 
tischer Hinsicht stütze ich mich dabei auf die phänomenologische Analyse der Strukturen der Lebenswelt durch Alfred Schütz (Schütz/ Luckmann 2003), die ich immer als »(Proto-)Theorie des Verstehens« (Eberle 1984, 2000) interpretiert habe und auch für geeignet halte, Sinndeutungsprozesse bei exotischer Fremdheit zu analysieren.

Die formalen Strukturen der Lebenswelt bilden eine Art Matrix, die sich als tertium comparationis nicht nur zum Vergleich historischer und soziologischer Daten (Luckmann 1973), sondern - wie Stubar (2005) ausführlich aufgezeigt hat - auch für die Analyse kultureller Differenzen eignet. Sie hypostasieren nicht eine bestimmte Kulturform zu einem universellen Deutungsschema, sondern stellen eine konstitutionsanalytisch gewonnene Deskriptionssprache zur Verfügung, die sowohl die Andersartigkeit und Differenz als auch die Gleichheit und Ähnlichkeit von Kulturformen transparent macht (Srubar 2005: 167-169). Sie bilden eine Protosoziologie (Luckmann 1973) und Protohermeneutik (Soeffner 1999), die offen bleibt für eine Vielfalt verschiedener theoretischer Ansätze und Zugangsweisen. Den Symbolen und Zeichensystemen, vorab der Sprache, kommt dabei eine herausragende Bedeutung zu. Das Konzept der Vielschichtigkeit der Lebenswelt legt dabei nahe, auf der sprachlichen Ebene immer von einer Vielzahl heterogener Semantiken auszugehen und diese komparativ zu erforschen (Srubar 2005: 169). Im Unterschied zu den sprachreduktionistischen Ansätzen geht die Kònstitutionstheorie der Lebenswelt allerdings davon aus, dass man die Analyse nicht erst auf der Ebene der Semiosis ansetzen darf, "ssondern von den tieferliegenden Ebenen des Bewusstseins, der Leiblichkeit und des Handelns ausgehen muss « (ebd.: 162). Das heißt mit andern Worten, dass mKulturs nicht rein semiotisch, d. h. nicht nur als Text begriffen werden kann«; Phänomene des »unmittelbaren leiblichen Kontakts wie Gewalt, Pflege, Sexualität etc.« bilden eine vorsprachliche, »asemiotische« Kommunikation (ebd.: 162).

Schütz hat sich mit den Sinndeutungsprozessen in all ihren Vielschichtigkeiten und Subtilitäten befasst, von den Akten der Selbstdeutung mit ihrer Transzendierung des Hier und Jetzt (den kleinen Transzendenzen) über das Fremdverstehen mittels Zeichen und Anzeichen (mittlere Transzendenzen) bis zu den nicht-alltäglichen, oft symbolisch appräsentierten Wirklichkeiten (den großen Transzendenzen). Hinsichtlich des Fremdverstehens geht der mundanphänomenologische Ansatz von Schütz von der Generalthesis des alter ego aus, nimmt die Existenz von Mitmenschen also als gegeben an. Aufgrund der unterschiedlichen milieu- und biographiespezifischen Wissensvorräte und Relevanzsysteme der Akteure ist Fremdverstehen stets nur approximativ möglich. Trotzdem geht Schütz nicht wie Luhmann von der Unwahrscheinlich- keit, sondern von der Wahrscheinlichkeit gelingenden Verstehens bzw. gelingender Kommunikation aus, legt also - wie es Knoblauch und Schnettler (2004) formulieren - nicht ein Alienitäts-, sondern ein Alteritätsmodell zugrunde. Im Alltag operieren Akteure denn auch erfolgreich mit der Annahme reziproker Perspektiven, welche die Idealisierung der Vertauschbarkeit der Standorte und die Idealisierung der Kongruenz der Relevanzsysteme umfasst. De facto werden sich die Relevanzsysteme meistens nur teilweise decken. Srubar (2005: 166 f.) schlägt vor, verschiedene Stufen dieser Kongruenz bzw. Reziprozitätserwartung gemäß folgender Typologie zu unterscheiden: a) die anthropologische Intersubjektivität ordnet das Gegenüber dem Typus »Mitmensch« zu; b) die soziale Intersubjektivität konstituiert sich in der face-to-face-Interaktion mit dem Gegenüber; c) die kulturelle Intersubjektivität bezieht sich auf die Deckung der Typik und Relevanzstruktur von Kulturformen. Nur Mitglieder derselben »in-group« können voneinander erwarten, dass sich alle drei Stufen decken.

\section{Affenmenschen?}

Beim ersten Treffen nach unserer Rückkehr in die Schweiz fragte uns eine hoch betagte Frau aus unserer Verwandtschaft: »Thr wart doch bei diesen Affenmenschen? Nun erzählt mal - wie war das denn? « Es war nichts Despektierliches in ihrer Frage, sondern bloße Neugier; es schwangen aber auch etwas Unbehagen und Furcht mit und gleichzeitig etwas Bewunderung, dass wir uns überhaupt zu diesen vorgewagt hatten. Der Ausdruck »Affenmensch« war mir zuvor nie begegnet, doch fand ich ihn unmittelbar plausibel: Wenn die Aborigines in Gruppen am Boden kauerten, mit ihren hageren, schlacksigen Körpern, ihren ungewöhnlich langen Armen, den tiefen Augenhöhlen und ihren gewölbten Backenknochen, scheinbar dumpf vor sich hin starrend und unverständliche Laute von sich gebend - da hatten wir auf Distanz in der Tat manchmal die Assoziation, eine Horde Menschenaffen vor uns zu haben. Das war selbstverständlich nur eine Assoziation aufgrund ihrer visuellen Erscheinung beim Kauern, denn wir wussten ja, dass sie Menschen waren - Menschen mit einer eigenen Kultur, einem hoch entwickelten Werkzeuggebrauch, einer eigenen Sprache, mannigfaltigen religiösen Symbolen, Zeremonien und Ritualen sowie einer ausdrucksstarken Kunst. Sie waren also durch eine ganze Reihe relevanter Merkmale von Tieren - hier: von Menschenaffen - abgrenzbar. »Affenmenschen « impliziert umgekehrt, dass sie zwar zur Gattung Menschen gehören, aber in einer nicht näher spezifizierten Weise Affen ähneln - sei es auf- 
grund visueller Assoziationen, sei es wegen einer behaupteten phylogenetischen Nähe zu den Menschenaffen oder sei es wegen ihrer pejorativ als »primitiv« oder »unzivilisiert « bezeichneten Kultur. Wie dem auch $\mathrm{sei}^{10}$ - die Rede von $»$ Affenmenschen« erinnert zum einen daran, dass die Aborigines von vielen Europäern lange mit Affen gleichgesetzt wurden, und zum anderen wirft sie die Frage auf, ob und inwiefern sich unsere Kommunikation mit ihnen tatsächlich von einer Mensch-TierKommunikation unterschied.

Die »anthropologische Intersubjektivität« ist durchaus eine zivilisatorische Errungenschaft, setzt sie doch die Zuordnung des Gegenübers zum Typus »Mitmensch« voraus. Seit den Anfängen menschlicher Zivilisation bis tief ins Dritte Reich (und mancherorts darüber hinaus) gab es mannigfaltige Kriterien, zwischen verschiedenen Sorten von Menschen zu unterscheiden und sie entsprechend unterschiedlich zu behandeln. Die Unterscheidung von Menschen und Barbaren oder zwischen Menschen und Untermenschen diente vielen Kriegen, Vertreibungen, Ausrottungen und insbesondere auch der Sklaverei als Legitimation. Es gab Typen von Menschen, die wurden von ihren Zeitgenossen schlimmer als Tiere behandelt. Auch die Kolonisatoren Australiens weigerten sich lange, die Aborigines als Menschen zu betrachten. Berichten zufolge wurden sie vom ersten Europäer, der mit ihnen in Kontakt kam - nämlich dem englischen Piraten William Dampier - als die »armseligsten Kreaturen auf der Erde« bezeichnet, die sich »von Tieren kaum unterscheiden« (Wegner 1996: 88). Der späteren Kolonialverwaltung fehlte jegliches Unrechtsbewusstsein, die fruchtbarsten Landstriche in Besitz zu nehmen, in Eigentumsparzellen einzuteilen und an die Siedler $z u$ verkaufen, welche dann Zäune darum herum errichteten - denn das Nomadenvolk der Aborigines kannte ja kein Eigentum an Grund und Boden, also nahm man ihnen - so die Argumentation - auch nichts weg. Mit der Kolonisierung setzte auch ein beispielloser Genozid ein, verursach durch eingeschleppte Krankheiten sowie systematische Vertreibungen und Kriege, und es sind zahllose Massaker, Vergewaltigungen und Versklavungsversuche überliefert (ebd.: 85-97). Schwarze zu erschießen, galt in aller Regel nicht als Verbrechen. Selbst australische Wissenschaftler behandelten diese wseltsamen und rohen Kreaturen, [... die] woanders ausstarben und höheren Lebensformen Platz machten«, bis in die 1920er Jahre mit Geringschätzigkeit (ebd.: 87). Von den Einwanderern bis hin zu den Regierungsberichten wurden die Eingeborenen bis

$10 »$ Menschenaffe« ist ein zoologischer terminus technicus, $»$ Affenmensch $\ll$ jedoch lediglich ein schwammiger und kaum mehr verwendeter Alltagsbegriff. weit ins 20. Jahrhundert despektierlich als »Wilde« bezeichnet. ${ }^{11}$ Und bis in die 1970er Jahre wurden Aborigines-Kinder ihren Familien entrissen und in Missionsschulen gesteckt, wo sie die englische Sprache und Kultur erlernen und damit »zivilisiert《 werden sollten. Erst 1975 wurde der $»$ Racial Discrimination Act« verabschiedet, der den institutionalisierten Rassismus gegen die Aborigines - kein Bürgerrecht, kein Pass, kein Wahlrecht, kein Recht, Land zu besitzen, kein Anspruch auf Mindestlöhne, kein Anspruch auf Sozialleistungen usw. - legalistisch beendete. ${ }^{12}$ Der alltägliche Rassismus lässt sich allerdings nicht per Dekret abschaffen und grassiert da und dort weiter.

Die Aborigines bilden ein illustratives Beispiel dafür, dass die anthropologische Intersubjektivität, also die Zuordnung des Gegenübers zum Typus »Mitmensch« nicht selbstverständlich ist. Die australischen Ureinwohner wurden von den Immigranten oft als Tiere oder tierähnlich betrachtet und behandelt, und wenn sie als Menschen angesehen wurden, dann meist nicht als ebenbürtige, sondern als minderwertige Menschen, nämlich als solche zweiter und dritter Klasse. Umgekehrt wissen wir aus soziologischen Studien, dass Menschen ihre Haustiere manchmal anthropomorphisieren, ihnen also allerlei menschliche Eigenschaften zuschreiben und entsprechend mit ihnen kommunizieren - was manchmal in der Aussage gipfelt, Tiere seien die besseren Menschen. Die Grenzziehungen zwischen Tier und Mensch wie auch die Unterscheidung von Menschen unterschiedlicher Wertigkeiten sind mit all ihren verschiedenen Konnotationen gesellschaftliche und als solche kulturelle Konstrukte, die in ihrem praktischen Verwendungszusammenhang empirisch erforscht werden müssen. Durch andere operative Prämissen in Perzeption und Interaktion verändert sich nämlich auch der lebensweltliche Konstitutionsprozess. Die mundanphänomenologische Konstitutionstheorie von Schütz läuft durch ihre sepoché der natürlichen Einstellung Gefahr, ontologische Setzungen der westlichen Lebenswelt als

11 Unter den deutschsprachigen Autoren vgl. etwa Hans Bertram (1995), der 1932 in Nordwest-Australien notlanden musste; allerdings substituierte er 1932 in Nordwest-Australien notlanden musste; allerdings substituierte er
später den Angst erregenden, anonymen Typus des »Wilden« ohne Umschweife, nachdem er von Aborigines gerettet wurde. Vgl. auch den österreichischen Taucher und Verhaltensforscher Hans Hass, der noch in den 1950er Jahren von »bösartigen Ureinwohnern« schrieb (Hass 1957: 72).

12 Während die Berechtigung, Land zu besitzen (meist im Kollektiveigentum) und medizinische Hilfe zu erhalten, längst überfällig war, wirkte sich der Anspruch auf Sozialleistungen eher verheerend aus, trugen sie doch als nicht-intendierte Konsequenz wesentlich dazu bei, die angestammte Lebensweise und Kultur zu zerstören. 
invariant zu erklären, die in andern kulturellen Kontexten anders geartet sind. ${ }^{13}$

\section{Die Irreziprozität der Perspektiven}

Was nun unsere eigenen Begegnungen mit Aborigines betrifft, haben wir in keinem Moment daran gezweifelt, Menschen vor uns zu haben. Die erste, allgemeinste Stufe, die anthropologische Intersubjektivität, war mithin gegeben. Nach Srubar (2005: 167) schlägt sie »sich nieder in den Annahmen, die mit der Vertauschbarkeit der Standorte verbunden sind und die von einer vergleichbaren humanen Sinnes-, Sprach- und Handlungsausstattung ausgehen.« Letzteres war für uns zweifellos der Fall. Allerdings hatten wir überhaupt nicht den Eindruck, mit diesen grundlegenden Annahmen allein sei die Vertauschbarkeit der Standorte bereits erfüllt. Es stellt sich daher die Frage, wie allgemein - auf welcher Generalisierungsstufe - die Idealisierung der Vertauschbarkeit der Standpunkte ausgelegt werden soll. Schütz definiert sie folgendermaßen: »Wäre ich dort, wo er jetzt ist, würde ich die Dinge in gleicher Perspektive, Distanz, Reichweite erfahren wie er; und wäre er hier, wo ich jetzt bin, würde er die Dinge in gleicher Perspektive erfahren wie ich « (Schütz/Luckmann 2003: 98). Diese Reduktion auf scheinbar rein räumliche Dimensionen wirkt allerdings etwas schal. Intuitiv ist die Vertauschbarkeit der Standpunkte doch dann optimal gegeben, wenn ich und das Gegenüber über weitgehend deckungsgleiche Wissensvorräte verfügen, wir also nicht nur derselben Gesellschaft, sondern derselben in-group angehören. Dann geht es beim Verständigungsprozess nur noch um die verschiedenen Perspektiven und die jeweiligen Relevanzsysteme, deren Unterschiedlichkeit je mit der Idealisierung der Vertauschbarkeit der Standpunkte und der Idealisierung der Kongruenz der Relevanzsysteme situativ überbrückt wird. Wenn die Idealisierung der Vertauschbarkeit der Standorte derart allgemein interpretiert würde, dass sie sich nur auf die räumliche Dimension bezöge und kulturelle Unterschiede keine Rolle spielten, dann könnte man sie ebenso gut auf ein tierisches Gegenüber anwenden: In Bezug auf räumliche Kalküle in einer Gefahrensituation, wenn ich im Dschungel einem Raubtier oder einem möglicherweise feindlich gesinnten Menschen gegenüberstehe, hilft mir die Annahme einer vergleichbaren humanen Sinnes-, Sprach- und Hand-

13 In dieselbe Richtung stößt die Kritik von Mehan/Wood (1975: 8-33), Schütz' Bezeichnung der Alltagswelt als >vornehmliche Wirklichkeit (paramount reality) treffe wohl auf die westliche Lebenswelt zu, nicht jedoch auf jene von südamerikanischen Indianern. lungsausstattung wohl nicht viel weiter. Wesentlich entscheidender wird sein, was ich über das typische Verhalten dieses konkreten Raubtiers bzw. über das typische Verhalten dieses konkreten menschlichen Gegenübers weiß. Die Vertauschbarkeit der Standpunkte ist umso besser gegeben, je mehr mir über die Weltwahrnehmung und das Verhaltensspektrum des Gegenübers bekannt ist - und das impliziert bei einem Menschen zwangsläufig ein Verständnis seiner Kultur. In der Tat definiert Schütz an anderer Stelle, dass die Idealisierung der Vertauschbarkeit der Standorte auch heiße, dass ich durch einen Platztausch die Dinge «in denselben typischen Aspekten sehe« (Schütz 1971: 13), was eo ipso eine Kenntnis der Sprache und der Weltsicht des Gegenübers voraussetzt. Bei unseren Begegnungen mit Aborigines hatten wir jedenfalls nie den Eindruck, unsere Standorte seien vertauschbar - zu wenig wussten wir von ihrer Lebenswelt. Die anthropologische Annahme, dass sie über eine analoge Sinnes-, Sprach- und Handlungsausstattung verfügten, weist uns allerdings den Weg, wie wir sie - im Unterschied zu jedem Tier - besser verstehen lernen könnten: durch das Erlernen ihrer Sprache, ihrer Lebensweise und ihrer Kultur.

Das Hauptproblem bei unseren Begegnungen mit Aborigines war in der Tat, dass wir thre Sprache nicht verstanden - und sie die unsere(n) nicht. Im pragmatischen Bemühen, uns mit ihnen zu verständigen, fühlten wir uns daher ähnlich »aufgeschmissen«, wie man es gegenüber Säuglingen oder Tieren sein kann: Man versucht die Anzeichen zu deuten, aber eine Verständigung ist unmöglich. Eltern fühlen sich oft ratlos, wenn ihr Säugling schreit, und rekurrieren wenn möglich auf typische Erfahrungswissensbestände, was angesichts der beobachtbaren körperlichen Symptome und Ausdrucksweisen in diesem Alter typischerweise der Fall sein könnte und was daher zu tun sei. Ähnlich geht es Tierhalter(inne)n, und in beiden Fällen finden sich in der Regel Ratgeber(innen). Uns ging es genauso: Wir fühlten uns immer wieder fundamental unsicher, was in den verschiedenen Situationen jeweils vor sich ging; daher suchten wir wiederholt den Rat von (weißen) Einheimischen, die sich mit Aborigines auskannten, um in Erfahrung zu bringen, wie diese oder jene konkrete Situation wohl zu interpretieren und was jeweils zu tun sei (z. B. im Aboriginal Art Centre).

Eine Knacknuss bildete daher auch die Idealisierung der Kongruenz der Relevanzsysteme, gemäß der wir es »als gegeben hinnehmen, dass Unterschiede der Auffassung und Auslegung, die sich aus der Verschiedenheit meiner und seiner biographischen Situation ergeben, ... für unsere gegenwärtigen praktischen Zwecke irrelevant sind « (Schütz/Luckmann 2003: 98). Bei exotischer Fremdheitserfahrung ist es jedoch gerade umgekehrt: Die Unterschiede in Auffassung und Auslegung sind dau- 
ernd relevant. Laufend stellt man sich die Frage, was das Gegenüber wohl meint und wie seine Ausdrucksweisen und die verwendeten Zeichen wohl $\mathrm{zu}$ interpretieren sind. Schon beim ersten Winken jener $\mathrm{Ab}$ origine-Frau unter dem Parkbaum in Darwin schossen mir sofort Gedanken durch den Kopf, was sie wohl damit meint und welches ihre möglichen Intentionen sind. Mein subjektiver Wissensvorrat stellte dazu zahlreiche Interpretationsressourcen zur Verfügung: einschlägige persönliche Erfahrungen im eigenen wie in anderen Kulturkreisen, aber auch unzählige Geschichten, die dem Alltag, der Literatur, Filmen oder Radio- und Fernsehberichten entstammen. Auch in den folgenden Begegnungen handelten wir stets unter großer Unsicherheit und Ungewissheit und suchten immer wieder Anschlussstellen und Bezugspunkte, die gemeinsam relevant sein könnten. Mit anderen Worten: Wir bemühten uns laufend, interaktiv eine Kongruenz der Relevanzsysteme zu erzeu gen. Obne gemeinsame Sprache gelang das nur sehr rudimentär, der Interaktionsfluss brach immer wieder zusammen, was bei ununterbrochener leiblicher Ko-Präsenz oft ein unangenehmes Gefühl in mir auf keimen ließ.

Als Zwischenfazit kann festgehalten werden, dass wir in diesen Situationen ausgeprägter Fremdheitserfahrung nicht mit einer Generalthesis der Reziprozität, sondern der Irreziprozität der Perspektiven operierten. Sowohl die Idealisierung der Vertauschbarkeit der Standorte als auch die Idealisierung der Kongruenz der Relevanzsysteme erwiesen sich als schlichtweg unpraktikabel. Die meisten unserer Alltagsroutinen brachen zusammen, jede Situation war problematisch, und der nichthinterfragte Horizont von Selbstverständlichkeiten war einem Gefühl von Unsicherheit, Ungewissheit und weitgehender Orientierungslosigkeit gewichen. Für die Situation exotischer Fremdheitserfahrung scheint daher Luhmanns Alienitätsmodell naheliegender zu sein als das Alteritätsmodell von Schütz: Die Wahrscheinlichkeit gelingender Verständigung ist minimal. Exotische Fremdheitserfahrungen sind allerdings lebensweltlich eher selten, weswegen es $\mathrm{m}$. E. opportuner ist, sie als Grenzerfahrungen innerhalb des Alteritätsmodells zu begreifen, statt normale Alltagssituationen mit dem Alienitätsmodell zu konzipieren.

\section{Das Sprachproblem}

Auch wenn unser Bemühen um Verständigung mit den Aborigines in pragmatischer Hinsicht vergleichbar problematisch war wie jene mit Säuglingen oder Tieren, liegt doch ein grundsätzlich anderer Sachverhalt vor: Sozialisierte und enkulturierte Menschen können sich mittels Zei- chen und Symbolen ausdrücken und in ihrer Sprache verständigen. Die ausgeprägte Fremdheitserfahrung konstituierte sich dadurch, dass wir keines dieser Zeichen und Symbole kannten und nichts von dem verstanden, was die Aborigines sagten, und nur wenig von dem, was sie taten. Die praktischen Schwierigkeiten, einen völlig Fremden zu verstehen, sind ebenso groß und ebenso schwer zu bewältigen wie die Probleme beim Versuch, ein Bébé oder ein Tier zu verstehen. Bei sozialisierten Menschen liegt indes weder ein Defizit an Kommunikationskompetenz (wie beim Säugling) noch ein Wesensunterschied (wie beim Tier) vor: Der Fremde ist grundsätzlich verstehbar, nur müssten wir seine - oder er unsere - Sprache beherrschen. Oder wir bräuchten einen Ubersetzer, der beide Sprachen spricht und versteht und die eine in die andere übertragen kann. Wir waren überrascht, wie schlecht die Englisch-Kenntnisse - Australiens Amtssprache - selbst unter den jungen Aborigines waren. Und selbst unser Guide, Gundumuk, konnte nicht als Übersetzerin fungieren. So versuchten wir also, im direkten Kontakt wenigstens einige grundsätzliche Interaktionsformen in Gang zu bringen. Ohne Sprachkenntnis blieben unsere Begegnungen auf die zweit Stufe der Reziprozitätserwartung beschränkt: auf die soziale Intersubjektivität. Srubar (2005: 167) definiert sie sals ein typisches Wissen, das mich soziale Beziehungen und Handlungen in ihrer einfachen Form er kennen/erwarten lässt (Kommunikationsabsicht, materialer Austausch, die Deixis der Geste, Über- und Unterordnung etc.), die in der face-toface-Beziehung manifest werden.« In der Tat konnten wir kooperativ eine ganze Reihe von Interaktionen in Gang setzen, welche dieselben Grundstrukturen aufwiesen, wie sie die ethnomethodologische Konversationsanalyse (Eberle 1997) für Gespräche in westlichen Ländern aufzeigte: Redezug-Wechsel, Frage-Antwort-Sequenzen, Austausch von Gesten, Koordination verbaler und non-verbaler Ausdrucksweisen usw. und auch der materiale Austausch von Nahrungsmitteln oder Zigaretten (bzw. das Geben und Nehmen derselben) gelang problemlos.

Die formalen Anforderungen, eine Interaktion in Gang zu setzen und in Gang zu halten, waren allerdings weniger schwierig zu bewältigen als das Etablieren einer gemeinsamen Referenz: Worüber konnten wir miteinander »ins Gespräch« kommen? Es lag nahe, dass wir uns auf ganz basale Dinge bezogen, und zwar zunächst auf das, was wir als universale anthropologische Konstanten betrachten: Jeder Mensch braucht Nahrung, Flüssigkeit, Schlaf und muss seine Notdurft verrichten. Wir verstehen daher aus eigener Erfahrung Grundbedürfnisse wie Hunger, Durst, Müdigkeit, den Drang, Wasser zu lassen usw., und wir finden sie in allen Kulturen. Die aufgezählten Grundbedürfnisse treffen zwar auch auf die meisten Tiere zu. Der Unterschied zum Tier liegt jedoch darin 
dass bei den Menschen all diese Verrichtungen nicht auf »natürliche« Art vorgenommen werden, sondern von kulturellen Regeln geleitet sind Dies gilt auch in Bezug auf weitere anthropologische Annahmen, mit denen wir operieren: Menschen können denken, fühlen, sprechen, lachen, weinen, träumen, sich etwas vorstellen, sich erinnern, sich auf mannigfaltige Art ausdrücken (und viele andere mehr). Je weniger wir die Sprache, Symbole und Normen des Gegenübers verstehen, desto mehr reduziert sich unser Verstehen auf das Auslegen äußerlicher Anzeichen.

Die erwähnten Grundbedürfnisse lassen sich in allen Kulturen verstehen, auch wenn ihre Befriedigung unterschiedlich geregelt ist. Auch Plaudern, Lachen und Weinen, Spielen und Jagen, Speer werfen, Feuer machen und Kochen, Schnitzen und Malen, Baby streicheln und Didgeridoo-Spielen lassen sich, zumindest an der Oberfläche, leicht nachvollziehen - zumal diese Tätigkeiten auch in unserer Sprache problemlos beschreibbar sind. Auf dieser Ebene gelang es uns denn auch immer wieder, eine Wir-Beziehung zu konstituieren, in synchroner gegenseitiger Bezogenheit und mit gemeinsamer Referenz. Allerdings blieb dies alles in jener Sinnschicht stecken, die Pike (1971) als etische von der emischen Ebene abzugrenzen versuchte: Das Verstehen beschränkte sich allein auf äußerlich beobachtbares Verhalten, ohne zum subjektiven Sinn vorzustoßen, den die Akteure mit ihren Handlungen verbanden - ja nicht einmal bis zum intersubjektiven Sinn, den die Handlungen für dieses Kollektiv hatten. Auch die kurzen Sätze der verbalen Kommunikation - »Aborigines nice people« - halfen nicht weiter, sondern hatten allein die Funktion, Interaktionen in Gang zu setzen und aufrechtzuerhalten sowie die Wir-Beziehung durch gegenseitige Wertschätzung zu stärken. Trotzdem: die unterstellten anthropologischen Universalien boten einen geeigneten Bezugspunkt, um trotz ausgeprägter Fremdheit auf einer basalen Ebene miteinander zu kommunizieren und ein Gefühl von grundsätzlicher Gemeinsamkeit herzustellen.

Als weitere Bezugspunkte anerboten sich jene zivilisatorischen Errungenschaften, die mittlerweile auch zum Alltag der Aborigines in Arnhem Land gehören und ihre Kultur überformen und hybridisièren: Autos, Motorräder, Strassen, Tankstellen, Häuser, Supermärkte, Abfall, Schulen, Staat, Polizei usw. Ohne Übersetzer und ohne die fremde Sprache zu verstehen, hatten wir allerdings trotz aller Sinndeutungsversuche nicht den Eindruck, von der Kultur der Aborigines und ibrer Lebensweise wirklich etwas begriffen zu haben. Von der dritten Stufe der Reziprozitätserwartung, der kulturellen Intersubjektivität, also dem »Bezug auf die je spezifischen, tiefer liegenden Deutungsschemata, die die je unterschiedlich geprägte, pragmatisch generierte Typik und die Relevanz- struktur von Kulturformen enthalten« (Srubar 2005: 167), blieben wir weit entfernt. Sie stellte jedoch, soviel war klar, die eigentlich relevante Schicht dar. Wir verspürten daher den Dirang, auch andere Quellen zu erschließen, um mehr über ihre Kultur zu erfahren.

\section{Die Überformung des Erlebten durch narrative Rahmungen}

Man kann nicht in tiefere Sinnschichten eines kulturellen Bedeutungsgewebes (Geertz 1983) eindringen, ohne das Sprach- und Symbolsystem zu erlernen. Dabei gibt es verschiedene Stufen: Je differenzierter beispielsweise der Wortschatz, desto besser. Den entscheidenden Unterschied markiert jedoch der Übergang vom passiven Verstehen einer Sprache zu ihrer aktiven Beherrschung: Man muss die Sprache-imGebrauch (Wittgenstein) kennen, die Geschichte der betreffenden Sprachgemeinschaft, die Konnotationen der Begriffe, die Sinnhorizonte sprachlicher Ausdrücke mit ihren emotionalen Werten und irrationalen Implikationen. Schütz (1972: 65) bringt dies prägnant auf den Punkt "Um eine Sprache frei als Ausdrucksschema zu beherrschen, muss man in ihr Liebesbriefe geschrieben haben: man muss in ihr beten und fluchen und die Dinge mit jeder nur möglichen Schattierung ausdrücken können, so wie es der Adressat und die Situation verlangen.« Bei den Aborigines kommt hinzu, dass sie eine hoch ausdifferenzierte Symbolwelt entwickelt haben, die teils in sprachlichen Versen und teils in Zeichnungen, Gemälden, bemalten Utensilien, aber auch in Ritualen und Zeremonien aktualisiert und tradiert wird. Um die Sinnwelt der Aborigines im persönlichen Zugang zu verstehen, muss man nicht nur mehrere Monate, sondern Jahre unter ihnen leben. Das haben zahlreiche Ethnologen in teilnehmender Beobachtung getan. Allerdings blieben auch ihnen jene Sphären, die nur Eingeweihten (Initiierten) zugänglich sind, in aller Regel verwehrt (vgl. Eberle 2001).

Der Rekurs auf Übersetzer und Informanten ist ein zweiter gangbarer, allerdings suboptimaler Weg. Mary und ihre Eltern haben uns viele Geschichten erzählt, Fragen beantwortet und Sachverhalte erklärt So besuchten wir den zweiten Stamm mit viel mehr Vorwissen, und wir konnten gar nicht anders, als unsere Beobachtungen entsprechend zu rahmen: der Mord, der Aufruhr im Stamm, die Schlägerei, die vielen essenden Verwandten, die christianisierte Gemeinschaft das alkohol freie Dorf und so weiter. Mit jedem weiteren Tag im Feld, jedem weiteren Gespräch mit der Lokalbevölkerung, jedem weiteren Artikel und jedem weiteren Buch über Geschichte, Kultur und Alltag der Aborigines wurde dieses Wissen reichhaltiger und differenzierter. Laufend kamen 
weitere Rahmenschichten (Goffman 1974) hinzu. So wurde es immer schwieriger, die eigenen Erfahrungen von diesem komplexen Verweisungshorizont abzukoppeln. Zunehmend fanden wir uns in der Situation eines Ethnographen der eigenen Kultur, der diese methodischsystematisch «befremden« muss (Hirschauer/Amann 1997), um nicht im eigenen Vorwissen und den eigenen lebensweltlichen Selbstverständlichkeiten gefangen zu bleiben. Rückblickend war es daher gerade für die vorliegende Fragestellung ein Glück, dass der vertraglich bestellte »Guide«, der uns zu den Aborigines hätte führen sollen und so viel über sie wusste, wegen eines organisatorischen Lapsus ortsabwesend war, und dass sein $»$ Ersatz«, die Gundumuk, so wenig Englisch sprach: Unser erster Feldaufenthalt war dadurch viel unbefangener, frei vom Rahmungswissen eines Informanten, und wir waren auf eine unmittelbare, unvermittelte Kontaktnahme und Beobachtung angewiesen. Mit allen Unsicherheiten und Ungewissheiten, denen man in einer derartigen Situation exotischer Fremdheitserfahrung ausgesetzt ist.

Ohne zusätzliche Rahmungswissensbestände wäre unser Verständnis allerdings schal, farblos und oberflächlich geblieben. So suchten wir auf viele Fragen schlüssige Antworten. Bei jedem Informanten stellte sich jedoch das Problem seiner Vertrauenswürdigkeit und der Gültigkeit seiner jeweiligen Aussagen. Heuristisch lassen sich folgende Abstufungen unterscheiden:

1. Die meist stereotypen Aussagen weißer ${ }^{14}$ Australier, die in den Städten leben und die Aborigines aus eigener Anschauung meist nur in Form heruntergekommener, in den Städten herumlungernder Alkoholiker kennen. Sie sagen meist mehr über die Sinnwelt der Informanten aus als über jene der Aborigines.

2. Das Rahmungswissen Weißer, die intensive eigene Erfahrungen mit Aborigines hatten, weil sie längere Zeit in unmittelbarer Nachbarschaft einer Aboriginal Community gelebt haben. Ihre Urteile beruhen meist auf Kontakten, die in einen pragmatischen Handlungszusammenhang eingebettet waren (z. B. ein Road House Betreiber, de sie sowohl als Arbeitskräfte wie auch als Konsumenten und Nachbarn erlebt hat). Manchmal überstieg ihr kulturelles Interesse pragmatische Motive (wie etwa bei Mary), manchmal war auch beides miteinander verkoppelt (wie z. B. bei einem jungen Lehrer, der $A b$ origines Künstler(inne)n den Umgang mit dem Computer beibringen wollte). Dieser Typ Informant ist sich stets bewusst, dass er nur von

14 Australien ist mittlerweile ein multikulturelles Land mit vielen Einwanderern aus asiatischen Ländern. Der Einfachheit halber beschränke ich hier die Differenzierung auf Schwarz/Weiß. einem bestimmten Stamm, nicht von den »Abos« generell, spricht, und seine Aussagen müssen stets im Licht ihrer (recht unterschiedlichen) Motive gewertet werden.

3. Ethnologische Forschungspublikationen. Sie beziehen sich ebenfalls stets auf einen bestimmten Stamm (von mehreren hundert), kommen in einem wissenschaftlichen Duktus daher und gelten in der Regel als seriös recherchiert (mit Ausnahme der frühen Publikationen).

4. Aboriginal Culture Centres: An verschiedenen Orten Australiens sind in intensiver Zusammenarbeit zwischen (weißen) Ethnologen und Repräsentanten der lokalen Eingeborenenbevölkerung Kulturzentren geschaffen worden, in denen mit Ausstellungsgegenständen, Schrifttafeln, Film-, Bild- und Tondokumenten sowie Live-Vorführungen von Tanzritualen, Didgeridoo-Spiel und Gesängen ink1. Speer- und Bumerang-Werfen usw. die Kultur eines AboriginesStamms vorgestellt wird. Diese Kultur-Zentren sind sicherlich die verlässlichste Quelle von Rahmungswissen, denn erstens sind (heutzutage geschulte) Aborigines selbst daran beteiligt gewesen, und zweitens sind diese Zentren erst in jüngster Zeit entstanden und beruhen auf dem fortgeschrittensten, theoretisch wie methodologisch reflektierten Stand ethnologischer Forschung in Australien. Andererseits richten sich diese Kulturzentren primär an die Touristen und sind, was das Niveau und die Differenziertheit der zur Verfügung gestellten Informationen angeht, entsprechend an den Bildungsstand und an die Aufmerksamkeitsspannweite des durchschnittlichen Touristen angepasst.

Solche Abstufungen kristallisieren sich erst in der systematischen Reflexion über die Validität der empirischen Daten heraus. Im Alltag wie beim Feldaufenthalt vermischten sie sich zu einem unübersichtlichen Meer und schwer entwirrbaren Gewebe von Informationen. Zudem operierten wir auch weiterhin mit zahllosen, zur Selbstverständlichkeit geronnenen Interpretationsschemata aus der eigenen Herkunftskultur. So entstanden immer wieder neue Verweisungszusammenhänge, die das Beobachtete in ein anderes Licht stellten oder einer Interpretation weitere Schattierungen und zusätzliche Varianten hinzufügten: Die Berichte und Geschichten überformten das selbst Erlebte und konstituierten immer wieder neue Erfahrungszusammenhänge. Die einzelnen Bestände von Rahmungswissen wiesen ganz unterschiedliche Grade von Konkretheit bzw. Abstraktheit, Personalisierung bzw. Anonymisierung, Partikularität vs. Generalisierung auf. Ihre Gültigkeit und Plausibilität waren oft schwer zu bestimmen, also behandelten wir sie als mögliche Erfahrungskonstruktionen. Schon die einfache, sehr generalisierte Aussage, 
(sämtliche) Aborigines seien schüchtern und scheu, eröffnete uns beispielsweise einen völlig frischen Blick auf unsere Begegnungen mit ihnen und auf ihr »komisches《 Verhalten bei der Begrüßung und danach Sie waren nicht arrogant und behandelten uns nicht wie Luft, sondern sie waren schüchtern und zurückhaltend.

Diese Interpretation plausibilisierte sich bei zahllosen weiteren $\mathrm{Be}-$ gegnungen mit Angehörigen ganz unterschiedlicher Stämme. Und sie erinnerte uns daran, dass auch die nordamerikanischen Indianer uns seinerzeit nicht in die Augen geblickt hatten - der direkte Blickkontakt war bei ihnen tabu. Auch die Berichte weißer Australier, welche eine Aborigines Community als Nachbarn hatten, erhellten uns viele Aspekte auf plausible Weise - vor allem in Bezug auf die wahrgenommenen kulturellen Unterschiede sowie die Art der Beziehungen, die sie untereinander pflegten. Selbst die Klischees australischer Stadtbewohner erachteten wir nicht einfach als wertlos, beschrieben doch die Berichte und Urteile eines jeden Informanten ein Stück soziale Wirklichkeit aus seiner jeweiligen Perspektive. Selbstkritisch ist beizufügen, dass auch der wissenschaftliche Beobachter vor Klischees nicht gefeit ist, wenn er im Rahmen eines pragmatischen Handlungszusammenhangs zur Perspektive der natürlichen Einstellung wechselt. Kritischer Hinterfragung bedürfen daher sowohl die Beschreibungen und Deutungen von Ethnologen als auch die eigenen generalisierten wissenschaftlichen Konzepte aus der eigenen Herkunftskultur, wie z. B. »Regelverstöße«, »Normenkonformität« und »Devianz«, mit denen man schnell zur Hand ist. Zwar erlauben sie - wie Klischees - die rasche Typisierung von Beobachtetem; doch wie steht es um ihre Adäquanz in Bezug auf die lokalen Sinnkonstruktionen faktischer Handlungszusammenhänge? Ohne ein vertieftes Sprach- und Kulturverständnis müssen solche Fragen offen bleiben. $\mathrm{Ob}$ die Perspektive der Aborigines ${ }^{15}$ in der australischen und wissenschaftlichen Öffentlichkeit künftig besser zum Tragen kommen wird, bleibt abzuwarten. Jedenfalls wird in den Aboriginal Culture Centres berichtet dass man mit den Darstellungen der weißen Ethnologen nicht zufrieden sei, seien ihre Publikationen doch von vielen Missverständnissen gespickt. In Zukunft - so das Versprechen - würden die Aborigines ihre Kultur selbst - und authentischer! - darstellen.

15 Leider begegneten wir - außer in den Culture Centers - keinem einzigen Aboriginal, der ein elaboriertes Englisch gesprochen und sich als Informant geeignet hätte.

\section{Die Relativität der Fremdheitserfahrung}

Die Unüberwindbarkeit kultureller Differenz - das Thema dieses Buch - ist bei exotischer Fremdheitserfahrung besonders eklatant. Verstehen und Verständigung sind nur auf einer sehr basalen, oberflächlichen Ebene möglich, und das immer nur durch die Brille der eigenen kulturellen Prägung hindurch. Srubars Typologie unterschiedlicher Stufen der Reziprozitätserwartung erwies sich als geeignet, einige Aspekte der Sinndeutungsprozesse näher zu betrachten. Sie soll nun auch die zusammenfassende und abschließende Betrachtung leiten.

Die Unterscheidung von anthropologischer, sozialer und kultureller Intersubjektivität resultiert aus der pragmatischen Konstitutionstheorie welche die Einheit der Lebenswelt und die Differenz von Kulturforme unterscheidet (Srubar 2005: 157). »Kultur« als »semantisch objektiviertes und pragmatisch materialisiertes Wissen« beruht auf Bewusstseinsprozessen, deren invariante Grundstruktur phänomenologisch beschrie ben werden kann (ebd.). »Die gleichen Mechanismen, durch die alle Kulturformen hervorgebracht werden (Identität), bewirken auch ihre Andersartigkeit (Differenz)« (ebd.: 155). Die »Strukturen der Lebenswelt« eignen sich in der Tat, im Kulturvergleich als tertium comparationis zu fungieren. Wie wir gesehen haben, laufen die mundanphänomenologischen Analysen von Schütz mit ihrer 〉epoché< der natürlichen Einstellung indes auch Gefahr, gewisse ontologische Setzungen mitzutransportieren, die kulturellen Ursprungs sind. Gerade die Aborigines und ihre Geschichte mit den europäischen Immigranten bilden ein illustratives Beispiel, dass Anthropologie und Kultur nicht leicht auseinander zu halten sind: Die Unterscheidung von Mensch und Tier oder von Mensch und Untermensch sind kulturspezifische Konstruktionen, und die Zuordnung zur einen oder andern Kategorie erfolgt in gesellschaftlichen Prozessen. Mit welchen anthropologischen Annahmen im Einzelnen operiert wird, ist daher immer auch eine empirische Frage.

Die grundlegende Stufe der anthropologischen Intersubjektivität, die von der gegenseitigen Zuordnung zum Typus Mit-Mensch und der gegenseitigen Zuschreibung einer vergleichbaren humanen Sinnes-, Sprach- und Handlungsausstattung ausgeht, ist indes heutzutage wohl in aller Regel gegeben - wie auch bei unseren Begegnungen mit Aborigines. Sie lässt sich auch, wie dargestellt, mit weiteren anthropologischen Annahmen anreichern, die für den Großteil der heutigen Weltbevölkerung weitgehend konsensfähig sein dürften. ${ }^{16}$ Welche Anteile dabei der

16 Berger und Luckmann (1970) haben in ihrer Wissenssoziologie die Schütz'sche Lebensanalyse auch auf theoretischer Ebene mit der philosophischen Anthropologie Gehlens und Plessners kombiniert. 
»Natur« des Menschen und welche einer globalisierten »Kultur« zuzuschreiben sind, kann hier dahingestellt bleiben. Auch die zweite Stufe der Reziprozitätserwartung, die soziale Intersubjektivität, bezieht sich auf derart grundlegende Merkmale sozialer Interaktion, dass ohne sie ein sozialer Austausch gar nicht zustande kommen könnte. Zwischenmenschliche Kommunikationen auf dieser Stufe bleiben denn auch, wie aufgezeigt, sehr rudimentär. Solange wir die Sprache des Gegenübers nicht beherrschen, bleiben auch unsere Beobachtungen notgedrungen oberflächlich, da auf Anzeichen beschränkt. Obwohl unsere Interpretationen von den Deutungsmustern unserer Herkunftskultur angeleitet werden, also durchaus semiotisch organisiert sind, können wir den Sinn, den die Handlungen für die anderen Akteure haben, nicht erschließen. Erst die kulturelle Intersubjektivität eröffnet uns den Zugang zur Sinnwelt des Gegenübers, indem sie sich an Typik und Relevanzstruktur der vorfindlichen Kulturformen orientiert. Zu diesen ist indes kein direkter Zugang möglich obne die nötige Sprachkompetenz, sei es die eigene oder jene eines Übersetzers. Einen indirekten Zugang bieten Geschichten, die man aus unterschiedlichsten Quellen erfährt.

Erst auf der Ebene kultureller Intersubjektivität stellt sich das Problem der Überwindbarkeit oder Unüberwindbarkeit kultureller Differenzen. Und zwar bereits auf sprachlicher Ebene: Wer verschiedene Sprachen spricht, weiß um die vielen lexikalischen und stilistischen Besonderheiten, die es oft verunmöglichen, Aussagen eins zu eins zu übersetzen. Andererseits gelingt es Sprachvirtuosen aber doch immer wieder, selbst schwierige literarische Texte einigermaßen stimmig in eine andere Sprache zu übertragen. Probleme der interkulturellen Kommunikation gibt es heutzutage auch innerhalb desselben Sprachraums, einerseits aufgrund der inneren Ausdifferenzierung und Pluralisierung moderner Gesellschaften (Gross 1994; Knoblauch 2005), andererseits infolge kultureller Überlagerungs- und Hybridisierungsprozesse im Rahmen der zunehmenden Globalisierung (Reckwitz 2001). Dabei mag es ebenfalls milieuspezifische Sprachcodes und Semantiken geben, vornehmlich geht es aber um Lebensformen. Im Fall unserer Begegnungen mit Aborigines ging es indessen nicht primär um kulturelle Differenzen, sondern um Fremdheitserfahrung. In der vis-à-vis-Situation hatten wir lediglich Zugang zur etischen Schicht ihrer Kultur. Das ist, wie wir gesehen haben, schon eine ganze Menge: Ich kann beschreiben, in was für Hütten sie leben, wie sie ihren Alltag organisieren, wie sie jagen, kochen und essen, wie sie Didgeridoos produzieren, bemalen und spielen usw. Aber man wird sich laufend der Tatsache bewusst, von der emischen Ebene ihrer Kultur nichts begriffen zu haben.
»Exotisch« war diese Fremdheitserfahrung insofern, als dass wir das Gefühl hatten, in einer völlig anderen Welt zu sein, die uns völlig fremd war und zu der wir nur schwer einen verstehenden Zugang fanden. Daher operierten wir nicht primär mit der Generalthesis der Reziprozität, sondern der Irreziprozität der Perspektiven. Wenn man allerdings die Bewusstseinsakte in ihrem zeitlichen Strom betrachtet, oszillierten die operativen Annahmen zwischen Reziprozität und Irreziprozität der Perspektiven je nach Situation: Richtet man den Blick auf die kulturelle Differenz, auf das Fremde und Unverständliche, dann operiert man mit der Annahme der Irreziprozität; hat man demgegenüber das Gemeinsame, Verbindende im Auge - beispielsweise die gegenseitige Neugier und die momentane Bereitschaft, zusammen zu sein, miteinander zu kommunizieren oder etwas gemeinsam zu unternehmen -, dann unterstellt man eine grundsätzliche Reziprozität. Die Annahme der Irreziprozität der Perspektiven hatte natürlich auch mit der spezifischen Situation unserer Begegnung zu tun: Wir kamen als Touristen zu diesen Stämmen, für einen kurzen Aufenthalt; wir waren nicht gekommen, um längere Zeit mit ihnen zu leben. Sonst hätten wir nämlich viel mehr mitbekommen, auch ohne Sprachkenntnis, allein aus dem pragmatischen Arbeitsund Kooperationszusammenhang heraus. Doch selbst diese Situation war in weitere gesellschaftliche Kontexte ${ }^{17}$ eingebettet, die für unser Erleben direkte Relevanz hatten: So hatten wir bei unseren Besuchen bei diesen Stämmen in keinem Moment Angst, dass wir uns einer unkalkulierbaren Gefahr ausgesetzt hätten, und selbst wenn uns einige Gesichter zuweilen eher grimmig vorkamen, fürchteten wir uns nicht vor plötzlich ausbrechenden Aggressionen. Dies beruhte allein auf unserem Vertrauen in das Funktionieren übergreifender gesellschaftlicher Mechanismen: Wenn Fremde sich bei diesen Stämmen in Gefahr begäben, hätte uns vorher sicherlich jemand gewarnt. Umgekehrt waren sich zweifello sämtliche Angehörigen des jeweiligen Stammes klar darüber, dass die Behörden des »weißen« Australiens keine Tat ungesühnt ließen, die uns Schaden an Leib und Leben zugefügt hätte. Die soziale Kontrolle bilde mittlerweile ein weltumspannendes Netz und reicht auch bis in die entlegensten Gebiete und zu den abgelegensten Stämmen. So war auf einer ganz grundsätzlichen, aber sehr existenziellen Ebene eine Kongruenz der Relevanzsysteme mit hoher Wahrscheinlichkeit sichergestellt.

So exotisch unsere Fremdheitserfahrung war - wir verstanden letztlich doch eine ganze Menge. Sie muss daher auch relativiert werden und zwar in zweifacher Hinsicht: Erstens bestand sie allein in einer kulturellen Fremdheitserfahrung. Im Übrigen operierten wir sowohl mit den

17 Vgl. dazu Knoblauch $(1995,2005)$. 
Annahmen der anthropologischen als auch der sozialen Intersubjektivität: Es stand für uns außer Zweifel, dass wir uns unter Menschen befanden, mit denen wir grundsätzlich interagieren konnten. Die Fremdheit der Aborigines unterschied sich daher essentiell von der Figur des wmaximal Fremden«, bei dem es sich um ein nonhumanes Gegenüber handelt (Schetsche 2004a, b) ${ }^{18}$ Zweitens war die Fremdheitserfahrung auch insofern relativ, als sie sich allein auf die emische Ebene der Kultur bezog; auf der etischen Ebene haben wir relativ viel verstanden. Besónders ausgeprägt war die Fremdheit beim Besuch des ersten Stamms. In der Folge wurde sie laufend reduziert - durch jedes zusätzliche Erlebnis und jede ncue Geschichte, die unsere Erlebnisse in weitere Sinnzusammenhänge einstellte und als Erfahrungen neu rahmte. Jeder Ethnograph weiß, wie sich persönliche Beobachtungen und narrative Rahmungen zu einem schwer entwirrbaren Amalgam vermischen. Das Erlebnis maximaler kultureller Fremdheit beschränkte sich genau genommen auf unsere allerersten Begegnungen und transformierte sich im Kontext neuer Sinnkonstruktionen zunehmend zu einer relativen Fremdheit, die mit zahlreichen vertrauten Elementen durchmischt war. Der Verweisungshorizont wurde fortwährend komplexer, und je relativer die Fremdheitserfahrung wurde, desto deutlicher konstituierten sich die Konturen kultureller Differenzen.

\section{Literatur}

Berger, Peter L./Luckmann, Thomas (1970): Die gesellschaftliche Konstruktion der Wirklichkeit. Eine Theorie der Wissenssoziologie, Frankfurt/M.: Fischer

Bertram, Hans (1995 [1933]): Flug in die Hölle. Mein australisches Abenteuer, Frankfurt/Berlin: Ullstein

DUDEN (1990): Das Fremdwörterbuch, Mannheim et al.: Dudenverlag

Eberle, Thomas S. (1984): Sinnkonstitution in Alltag und Wissenschaft.

Der Beitrag der Phänomenologie an die Methodologie der Sozialwissenschaften, Bern/Stuttgart: Haupt

Eberle, Thomas S. (1997): Ethnomethodologische Konversationsanalyse, in: Hitzler, Ronald/Honer, Anne (Hg.), Sozialwissenschaftliche Hermeneutik, Opladen: Leske+Budrich, S. 245-279

Eberle, Thomas S. (2000): Lebensweltanalyse und Handlungstheorie. Beiträge zur Verstehenden Soziologie, Konstanz: UVK

18 Vgl. etwa Hitzlers (2004) Analyse der Begegnungen Ripleys mit der »Alien«-Spezies.
Eberle, Thomas S. (2001): Are the Great Warriors dying? Aborigines in der Multioptionsgesellschaft, in: Brosziewski, Achim/Eberle, Thomas S./Maeder, Christoph (Hg.), Moderne Zeiten. Reflexionen zur Multioptionsgesellschaft, Konstanz: UVK, S. 25-42

Geertz, Clifford (1983): Dichte Beschreibung. Beiträge zum Verstehen kultureller Systeme, Frankfurt/M.: Suhrkamp

Goffman, Erving (1974): Rahmenanalyse. Ein Versuch über die Organisation von Alltagserfahrung, Frankfurt/M.: Suhrkamp

Gross, Peter (1994): Die Multioptionsgesellschaft, Frankfurt/M.: Suhrkamp

Hass, Hans (1957): Wir kommen aus dem Meer. Forschungen und Abenteuer mit der Xarifa, Berlin: Ullstein

Hirschauer, Stefan/Amann, Klaus (Hg.) (1997): Die Befremdung der eigenen Kultur. Zur ethnographischen Herausforderung soziologischer Empirie, Frankfurt/M.: Suhrkamp

Hitzler, Ronald (2004): Ripleys Befremdung. Eine individualisierungstheoretische Deutung von »Alien Resurrection«, in: Schetsche, Michael (Hg.), Der maximal Fremde. Begegnungen mit dem Nichtmenschlichen und die Grenzen des Verstehens, Würzburg: Ergon, S. $113-124$

Hitzler, Ronald/Eberle, Thomas S. (2000): Phänomenologische Lebensweltanalyse, in: Flick, Uwe/Kardorff, Ernst von/Steinke, Ines (Hg.), Qualitative Forschung. Ein Handbuch, Reinbek: Rowohlt, S. 109118

Honer, Anne (1993): Lebensweltliche Ethnographie, Wiesbaden: DUV

Honer, Anne (2000): Lebensweltanalyse in der Ethnographie, in: Flick, Uwe/Kardorff, Ernst von/Steinke, Ines (Hg.), Qualitative Forschung. Ein Handbuch, Reinbek: Rowohlt S. 194-204

Knoblauch, Hubert (1995): Kommunikationskultur. Die kommunikative Konstruktion kultureller Kontexte, Berlin/New York: de Gruyter

Knoblauch, Hubert (2005): Die kommunikative Konstruktion kultureller Kontexte, in: Srubar, Ilja/Renn, Joachim/Wenzel, Ulrich (Hg.), Kulturen vergleichen. Sozial- und kulturwissenschaftliche Grundlagen und Kontroversen, Wiesbaden: VS, S. 172-194

Knoblauch, Hubert/Schnettler, Bernt (2004): »Postsozialität«, Alterität und Alienität, in: Schetsche, Michael (Hg.), Der maximal Fremde. Begegnungen mit dem Nichtmenschlichen und die Grenzen des Verstehens, Würzburg: Ergon, S. 23-41

Luckmann, Thomas (1973): Philosophie, Sozialwissenschaft und Alltagsleben, in: Soziale Welt, 24, S. 137-168

Mehan, Hugh/Wood, Houston (1975): The Reality of Ethnomethodology, New York: Wiley 
Pike, Kenneth L. (1971): Language in Relation to a Unified Theory of the Structure of Human Behavior, Den Haag: Mouton

Reckwitz, Andreas (2001): Multikulturalismustheorien und der Kulturbegriff. Vom Homogenitätsmodell zum Modell kultureller Interferenzen, in: Berliner Journal für Soziologie, 11 (2), S. 179-200

Schetsche, Michael (Hg.) (2004a): Der maximal Fremde. Begegnungen mit dem Nichtmenschlichen und die Grenzen des Verstehens, Würzburg: Ergon

Schetsche, Michael (2004b): Der maximal Fremde - eine Hinführung, in: ders. (Hg.), Der maximal Fremde. Begegnungen mit dem Nichtmenschlichen und die Grenzen des Verstehens, Würzburg: Ergon, S. 13-21

Schütz, Alfred (1971): Wissenschaftliche Interpretationen und Alltagsverständnis menschlichen Handelns, in: ders., Gesammelte Aufsätze, Bd. 1: Das Problem der sozialen Wirklichkeit, Den Haag: Nijhoff, S. 3-54

Schütz, Alfred (1972): Der Fremde, in: ders., Gesammelte Aufsätze, Bd. 2: Studien zur soziologischen Theorie, Den Haag: Nijhoff, S. 53-69

Schütz, Alfred/Luckmann, Thomas (2003): Strukturen der Lebenswelt, Konstanz: UVK

Soeffner, Hans-Georg (1999): „Strukturen der Lebenswelt« - ein Kommentar, in: Hitzler, Ronald/Reichertz, Jo/Schröer, Norbert (Hg.) Hermeneutische Wissenssoziologie, Konstanz: UVK, S. 29-37

Srubar, Ilja/Renn, Joachim/Wenzel, Ulrich (Hg.) (2005): Kulturen vergleichen. Sozial- und kulturwissenschaftliche Grundlagen und Kontroversen, Wiesbaden: VS

Srubar, Ilja (2005): Die pragmatische Lebenswelttheorie als Grundlage interkulturellen Vergleichs, in: Srubar, Ilja/Renn, Joachim/Wenzel, Ulrich (Hg.), Kulturen vergleichen. Sozial- und kulturwissenschaftliche Grundlagen und Kontroversen, Wiesbaden: VS, S. 151-17

Wegner, Dirk (1996): Australien. Ein Reisebuch in den Alltag, Reinbek: Rowohlt

\title{
Hegemoniale Moral: \\ Die Einebnung kultureller Differenz in der Debatte um den 3. Golfkrieg
}

\author{
ANDREAS GÖTTLICH
}

Der nachstehende Beitrag nimmt seinen Ausgang von einer empirischen Fallstudie zum öffentlichen Diskurs ${ }^{1}$ um die moralische Legitimität des Dritten Golfkrieges im Frühjahr 2003. Ziel derselben ist es, eine spezifisch wissenssoziologische Erklärung für die augenscheinliche Unvereinbarkeit zwischen den Argumentationen der Gegner und der Befürworter des militärischen Eingreifens im Irak zu finden. Gesucht wird diese Erklärung in den unterschiedlichen Deutungsmustern, welche die Diskussionsparteien bei ihren jeweiligen Situationsdefinitionen anwendeten, wobei ein besonderes Augenmerk der Figur des Perspektivenwechsels gilt, die im moralischen Kontext eine Überwindung partikularer Interessen bewerkstelligen soll. Im Falle der Irak-Debatte hatte die Anwendung dieser Denkfigur kulturelle Grenzen zu überwinden, insofern es galt, aus westlicher Sicht die Interessenlage der irakischen Zivilbevölkerung zu beurteilen.

Der gewählte wissenssoziologische Ansatz vermag zu zeigen, dass hierbei spezifische Urteilsmuster der je eigenen Kultur zur Anwendung kamen, dass mithin kulturelle Differenz weniger überwunden, denn vielmehr überdeckt wurde. Im Text wird diese, aus der empirischen Analyse sich ergebende These anhand des konkreten Beispiels der Argumentationsfigur der US-amerikanischen Regierung illustriert. Hierauf

1 Die Begriffe Debatte, Diskurs oder auch Diskussion werden im Folgenden weitgehend synonym verwendet. 
Jochen Dremer, Peter Stegmaier (Hg.)

Zur Unüberwindbarkeit kultureller Differenz

Grundlagentheoretische Reflexionen

\section{[transcript]}




\section{Inhalt}

JOCHEN DREHER UND PETER STEGMAIER

Einleitende Bemerkungen: `Kulturelle Differenz $\prec$ aus

wissenssoziologischer Sicht

Bibliografische Information der Deutschen Bibliothek

Die Deutsche Bibliothek verzeichnet diese Publikation in der Deutschen Nationalbibliografie; detaillierte bibliografische Daten sind im Internet über http://dnb.ddb.de abrufbar.

\section{(C) 2007 transcript Verlag, Bielefeld}

Die Verwertung der Texte und Bilder ist ohne Zustimmung des Verlages urheberrechtswidrig und strafbar. Das gilt auch für Vervielfältigungen,

Übersetzungen, Mikroverfilmungen und für die Verarbeitung mit elektronischen Systemen.

Umschlaggestaltung: Kordula Röckenhaus, Bielefeld

Lektorat \& Satz: Jochen Dreher und Peter Stegmaier

Druck: Majuskel Medienproduktion GmbH, Wetzlar

ISBN 978-3-89942-477-5

Gedruckt auf alterungsbeständigem Papier mit chlorfrei gebleichtem Zellstoff.

Besuchen Sie uns im Internet: http://vruw.transcript-verlag.de

Bitte fordern Sie unser Gesamtverzeichnis und andere Broschüren an unter: info@transcript-verlag.de

\section{Konstitution und Konstruktion skultureller Differenz}

HUBERT KNOBLAUCH

Kultur, die soziale Konstruktion, das Fremde und das Andere

ILJA SRUBAR

Transdifferenz, Kulturhermeneutik und alltägliches Übersetzen:

Die soziologische Perspektive

JOACHIM RENN

Vertraute Fremdheit und desperate Vergemeinschaftung -

Ethnizität und die doppelte Normalisierung kultureller Differenz in der Moderne

HANS-GEORG SOEFFNER

Methodologischer Kosmopolitismus - Die Erhaltung kultureller

Vielfalt trotz wirtschaftlicher und kultureller Globalisierung

SHINGO SHIMADA

Kulturelle Differenz und Probleme der Übersetzung 


\title{
Materiale und methodologische Analysen
}

\section{BARBARA ZIELKE}

Attributionen kultureller Fremdheit in der Psychotherapie:

Vom Kulturstandard zur kulturspezifischen Gegenübertragung

RONALD KURT

Indische Musik - Europäische Musik: Möglichkeiten und

Grenzen interkulturellen Verstehens

NORBERT SCHRÖER

Die dialogische Anverwandlung -

Zur Ausdeutung fremdkultureller Daten mit Hilfe von

>kulturvertrauten Co-Interpreten<

THOMAS S. EBERLE

Unter Aborigines: Reflexionen über eine exotische

Fremdheitserfahrung

\section{ANDREAS GÖTTLICH}

Hegemoniale Moral: Die Einebnung kultureller Differenz

in der Debatte um den 3. Golfkrieg

\section{Einleitende Bemerkungen: >Kulturelle Differenz< aus wissenssoziologischer Sicht}

\author{
JOCHEN DREHER UND PETER STEGMAIER
}

Was ist eigentlich darunter zu verstehen, wenn in einer globalen Welt von einem Aufeinandertreffen der Kulturen die Rede ist? Welche sozialen Prozesse laufen ab, wenn Konflikte zwischen den Kulturen oder gar Kulturkämpfe auftreten? Was wird postuliert, wenn umgekehrt der Ruf nach `Kosmopolitisierung rung von Kulturphänomenen laut wird? Auf diese und ähnliche Fragestellungen antworten die im Sammelband vertretenen Beiträge, die das Phänomen der `kulturellen Differenz` ausgehend von empirischen Erkenntnissen grundlagentheoretisch reflektieren. Es sind nicht »die Kulturen«, also die kulturellen Systeme selbst, die sich begegnen - sich begegnen können nur Menschen. Diese tragen immer schon die Erfahrungen des Andersseins gegenüber den begegnenden Individuen und deren sozialen Welten in sich. Verständigung ist allenfalls approximativ möglich. Viele Globalisierungstheorien setzen zu einseitig emphatisch bei der Wünschbarkeit von politisch-kultureller Verständigung und idealistisch überhöht bei kollektiven und übernationalen Fragen der Kosmopolitisierung an. Wir wollen das Augenmerk im vorliegenden Band primär auf das Verstehen richten.

Die Auseinandersetzung mit der Problematik des Kulturkontakts und der kulturellen Codierung sozialer Ungleichheit verlangt eine Analyse der Grundlagen jener sozialen Prozesse, in welchen Phänomene wie \Kulture und diesbezüglich insbesondere $\supset$ Fremdheit herausgebildet bzw. konstruiert werden. Ausgehend vom methodologischen Individualismus Max Webers muss die Perspektive des Subjekts ins Auge gefasst werden, um beschreiben zu können, mit welchen »Mechanismen« des 